\title{
American Capped Call Options on Dividend-Paying Assets
}

\author{
Mark Broadie \\ Columbia University \\ Jerome Detemple \\ McGill University and CIRANO
}

This article addresses the problem of valuing American call options with caps on dividendpaying assets. Since early exercise is allowed, the valuation problem requires the determination of optimal exercise policies. Options with two types of caps are analyzed constant caps and caps with a constant growth rate. For constant caps, it is optimal to exercise at the first time at which the underlying asset's price equals or exceeds the minimum of the cap and the optimal exercise boundary for the corresponding uncapped option. For caps that grow at a constant rate, the optimal exercise strategy can be specified by three endogenous parameters.

The valuation of American capped call options is a problem of theoretical as well as practical importance. Indeed, in the past few years several securities have been issued by financial institutions which include cap features combined with standard American call options. One example is the Mexican Index-Linked Euro Security, or "MILES." The MILES is an American

This paper was presented at the 1993 Derivative Securities Symposium, 1993 Western Finance Association Meetings, 1994 American Finance Association Meetings, Baruch College, and Université de Genève. We thank the participants of the seminars, Phelim Boyle, Bjorn Flesaker, and two anonymous referees for their comments. We are especially grateful to the editor, Chi-fu Huang, for detailed suggestions which have improved this paper. Address correspondence to Mark Broadie, 415 Uris Hall, Graduate School of Business, Columbia University, New York, NY 10027. 
call option on the dollar value of the Mexican stock index, the Bolsa Mexicana de Valores. The option is nonstandard because it has both a cap and an exercise period that is less than the full life of the option. The underlying asset is an index which involves dividend payments. The decision to exercise the option is under the control of the security holder.

Other examples of options with caps are the capped call (and put) options on the S\&P 100 and S\&P 500 indices that were introduced by the Chicago Board of Options Exchange (CBOE) in November, 1991. These capped index options combine a European exercise feature (the holder does not have the right to exercise prior to maturity) and an automatic exercise feature. The automatic exercise is triggered if the index value exceeds the cap at the close of the day. Flesaker (1992) discusses the design and valuation of capped index options. These options differ from the MILES because the holder of the option does not have any discretion In the exercise policy; i.e., they are not American options. Other examples of European capped call options include the range forward contract, collar loans, indexed notes, and index currency option notes [see Boyle and Turnbull (1989) for a description of these contracts].

The motivation for introducing capped options is clear. Written uncapped call options are inherently risky because of their unlimited liability. By way of contrast, capped call options have limited liability and are therefore attractive instruments to market for an issuer, or to hold short for an investor. Wide acceptance of these new securities in the marketplace, however, depends on a thorough understanding of their features and properties. Understanding valuation principles is important not only for pricing these new securities, but also for hedging the risks associated with taking positions in the securities. Because early exercise (i.e., before the option's maturity) may be beneficial with American capped call options, it is also essential to understand the structure of optimal exercise policies.

While these types of securities can be valued numerically by standard techniques, numerical results alone offer little explanation as to why certain exercise strategies are optimal. When standard binomial valuation procedures are applied to capped call options, the number of iterations required to obtain a desired level of accuracy is greatly increased compared to uncapped options. In this article, optimal exercise policies and valuation formulas are derived.

Options with caps were studied by Boyle and Turnbull (1989) Their article provides valuation formulas for European capped options as well as insights about early exercise for American capped options. The possibility of optimal early exercise with American capped 
call options was recognized by Boyle and Turnbull. They point out that when the underlying asset price substantially exceeds the cap, immediate exercise dominates a waiting policy.

Barrier options are related contracts which were studied in Cox and Rubinstein (1985) and more recently in Rubinstein and Reiner (1991). Barrier options combine a European-style exercise feature and an automatic exercise feature. The automatic exercise is triggered if the price of the underlying asset reaches the barrier (cap). The automatic exercise feature is slightly different than for CBOE's capped index options, where exercise can only occur at the close of a trading day. The combination of exercise features of barrier options -places them between European options and American options.

The presence of a cap on a call option complicates the valuation procedure. The floor on an option (i.e., the strike price) gives an incentive to exercise as late as possible. This is the source of the classical result that early exercise is suboptimal for American uncapped call options on non-dividend paying stocks. The American feature is worthless in this case, and American and European options have the same value. When the underlying asset pays dividends an incentive to exercise early is introduced. The optimal exercise boundary arises from the conflict between these two incentives. For standard American options without caps, the optimal exercise boundary can be written as the solution to an integral equation [see, e.g., Kim (1990) and Carr, Jarrow, and Myneni (1992)].

The introduction of a cap adds a further incentive to exercise early. On the surface, it seems that the cap could interact with the previous incentives to completely alter the form of the optimal exercise region. However, we show that the optimal exercise region is changed in a straightforward way. We show that the optimal exercise policy is to exercise at the first time at which the underlying asset's price equals or exceeds the minimum of the cap and of the optimal exercise boundary for the corresponding uncapped call option. For lowand non-dividend paying assets the optimal exercise policy simplifies to exercising at the cap. When the underlying asset price follows a geometric Brownian motion process with constant proportional dividends, an explicit valuation formula is given.

The valuation formula is then generalized to options with delayed exercise periods, that is, American options that cannot be exercised before a prespecified future date. The MILES contract has a delayed exercise period, which can be viewed as a time-varying or growing cap. In this case, the growing cap is a step function with a single jump. Growing caps may be preferred by investors since the upside potential increases over time. These caps may also be attractive to issuers who can accept a larger potential liability as time passes. A step function is 
an extreme form of a growing cap. We proceed to analyze caps that grow at a constant rate. For these caps we derive optimal exercise strategies and show that they can be completely characterized in terms of only three endogenous parameters.

The article is organized as follows. American capped call options with constant caps are analyzed in the next section. Results are given for finite and infinite maturity options. Section 2 extends the analysis to capped call options that have delayed exercise periods and to the MILES contract, that is, a capped call option on the dollar value of an index with a delayed exercise period. The case of caps that grow at a constant rate is considered in detail in Section 3. Section 4 provides numerical results comparing European capped calls to their American counterparts. A comparison of hedge ratios is also given. Conclusions and remarks on possible extensions of the model are given in Section 5. Proofs of results and some of the more lengthy formulas are collected in the Appendix.

\section{Valuation of American Capped Call Options}

We consider a class of derivative securities written on a dividendpaying underlying asset which may be interpreted as a stock or an index. The price of the underlying asset, $S_{t}$, satisfies the stochastic differential equation

$$
d S_{t}=S_{l}\left[(\mu-\delta) d t+\sigma d W_{t}\right],
$$

where $\mathbb{W} \equiv\left\{\mathbb{W}_{t}, \mathcal{F}_{t}: t \in[0, T]\right\}$ is a Brownian motion process and the coefficients $\mu, \delta$, and $\sigma$ are constants. Equation (1) implies that the stock price follows a geometric Brownian motion (lognormal) process with a constant dividend rate of $\delta$. We also assume that funds can be invested in a riskless money market account bearing a constant positive rate of interest denoted $r$.

Let $\boldsymbol{C}_{\boldsymbol{t}}^{\boldsymbol{L}}$ represent the value of an American capped call option at time $t$. The option has a strike price of $K$, a cap of $L$, and a maturity of $T$. Throughout the article, we assume that $L \geq K>0$. Exercise may take place, at the discretion of the owner of the security, at any date during the life of the option $[0, T]$. The payoff of the capped option when exercised at time $t$ is $\left(\left(S_{t} \wedge \boldsymbol{L}\right)-\boldsymbol{K}\right)^{+}$, where the operator $x \wedge y$ denotes $\min (\mathrm{x}, \mathrm{y})$ and $x+$ denotes $\max (\mathrm{x}, 0) .{ }^{1}$

\footnotetext{
'The payoff is assumed to be $\left((S, \cap D-K)^{+}\right.$even when the option is exercised out-of-the-money, that is, when $S_{i} \leq K$. This assumption is for expositional convenience only. It eliminates the need to describe exencise policles using separate cases for $\mathcal{S}_{t} \leq \boldsymbol{K}$ and $S_{\mathcal{H}}>\boldsymbol{K}$. In reality, the option would never be exercised when $\mathcal{S}_{\boldsymbol{f}} \leq \boldsymbol{K}$.
} 
Let $B_{t}$ denote the optimal exercise boundary for an uncapped call option with-the same maturity and strike price as the capped call option. That is, immediate exercise is optimal at time $\boldsymbol{t} \in[0, T]$ for all prices $S_{t} \geq B_{t}$. Integral equations which implicitly define $B_{t}$ are given in Kim (1990) and Carr, Jarrow, and Myneni (1992).

Theorem 1. Consider an American call option with exercise price $K$, cap equal to $L$, and maturity $T$. Let $\bar{B}$ idenote the optimal exercise boundary of the capped option. Then, fort $\in[0, T]$,

$$
B_{l}=L \wedge B_{\ell}
$$

That is, immediate exercise is optimal for the capped option if $\boldsymbol{S}_{\boldsymbol{t}} \geq \bar{B}_{\boldsymbol{t}}$ and suboptimal if $S_{t}<B_{t}$.

Because of the conflicting exercise incentives provided by a floor, cap, and dividends, the optimal exercise boundary might seem to be quite complicated. However, Theorem 1 shows that the optimal exercise boundary for a capped option has a simple relationship to the optimal exercise boundary for the corresponding uncapped option. It says that the optimal exercise time for the capped option is the minimum of the first time at which the price of the underlying asset attains or exceeds the value of the cap and the first time at which exercise of the uncapped option is optimal. The shaded area in Figure 1 illustrates an optimal exercise region, that is, the set of times and asset prices $\left(t, S_{t}\right)$ where it is optimal to exercise the capped call option.

In the case of no dividends, a simple arbitrage argument shows that a stronger result holds. Namely, for a continuous time-varying cap denoted $L_{t}$, it is not optimal to exercise at any time $t$ for which $S_{t}<L_{t}{ }^{2}$ This result holds even if the cap declines precipitously shortly after time t.

For small dividend rates with a constant cap the optimal exercise policy simplifies. Recall that $B_{t}$ is decreasing in $t$ and $B_{T}=$ $\max \{(r / \delta) K, K\}$. Hence, for dividend rates $\delta \leq r K / L, B_{t} \geq L$ for all $\boldsymbol{t} \in(0, \mathrm{~T}]$. In this case $B_{t}=L$, and the optimal exercise policy for the capped option is to exercise at the first time at which the price of the underlying asset equals or exceeds the value of the cap.

\footnotetext{
${ }^{2}$ Suppose an investor purchases one call, shorts one unit of the underlying asset, and invests the strike price in the riskless bond. If the value of the call were equal to the immediate exercise value, these transactions would have zero cost. Now, if the investor closes the position at the first hitting time of the time-depedent boundary or at maturity, the net cash flow is always nonnegative and is strictly positive with positive probability, which implies an arbitrage opportunity. Hence, no-arbitrage means that immediate exercise is not optimal for $S_{t}<L_{t}$.
} 


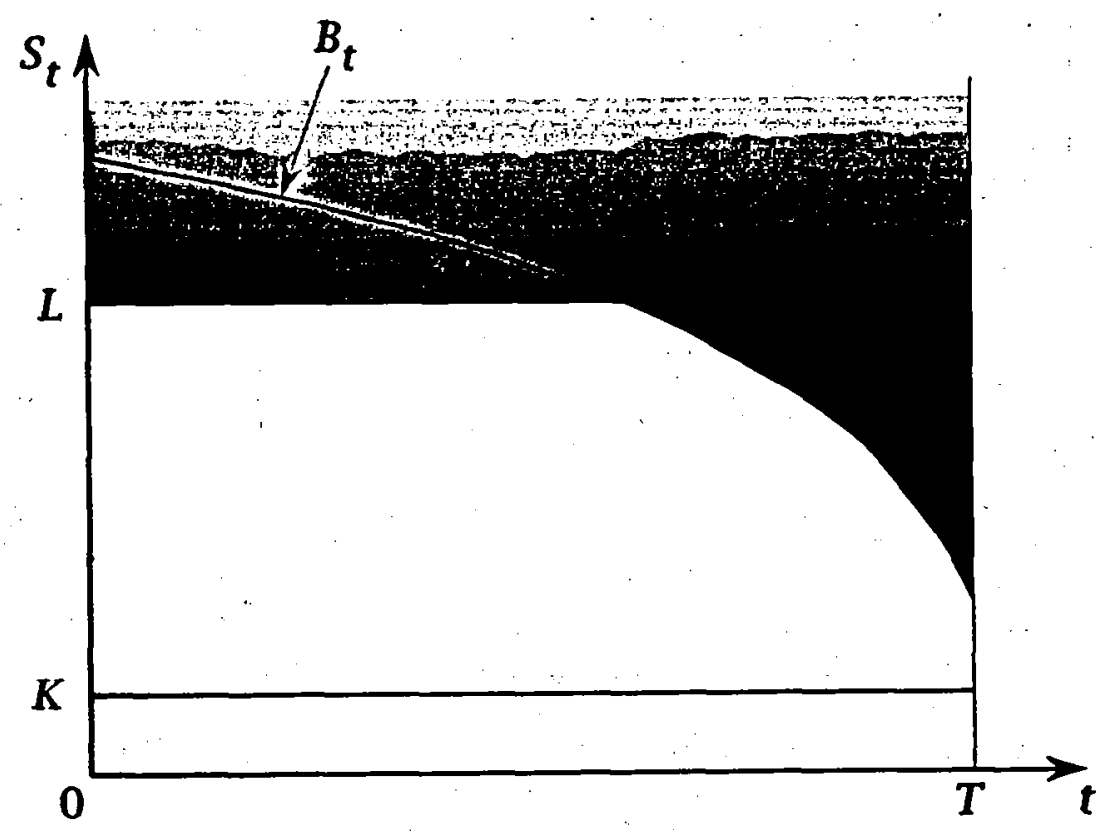

Figure 1

Optimal exercise region

Once the optimal exercise policy is known it is straightforward to derive a valuation formula for the American capped call option. We first consider the special case of an infinite maturity option. Recall that the optimal exercise boundary for an infinite maturity uncapped option is a constant, which we denote $\boldsymbol{B}_{\infty} .{ }^{3}$

\section{Corollary 1 (American capped call with infinite maturity).}

Consider an American capped call option with infinite maturity and payoff $\left(\left(S_{t} \wedge L\right)-K\right)^{+}$if exercised at time $t$. Then the optimal exercise boundary of the capped option is the constant $\boldsymbol{L} \wedge \boldsymbol{B}_{\infty}$. That is, the optimal exercise time is $\boldsymbol{\tau}=\inf \left\{\boldsymbol{v} \in[\boldsymbol{t}, \infty): \mathcal{S}_{\boldsymbol{v}} \geq \boldsymbol{L} \wedge B_{\infty}\right\}$.The value of the option at time $t$ for $S_{t}<L \wedge B_{\infty}$ is

$$
C_{t}^{L}\left(S_{t}, K, \infty\right)=\left(L \wedge B_{\infty}-K\right)\left(\frac{S_{t}}{L \wedge B_{\infty}}\right)^{2 \alpha / \sigma^{2}}
$$

where $\alpha$ is defined in (9) below. Furthermore, if the underlying asset

\footnotetext{
${ }^{3}$ An explicit expression for $B_{\infty}$ is $K(b+f) /\left(b+f-\sigma^{2}\right)$, where $b$ and $f$ are defined in $(\theta)$.
} 
pays no dividends, that is, $\delta=0$, then the option value simplifies to ${ }^{4}$

$$
C_{t}^{L}\left(S_{t}, K, \infty\right)=(L-K) \frac{S_{l}}{L}
$$

In both cases, for $S_{t} \geq L \wedge B_{\infty}$ the option value is $\left(S_{t} \wedge L\right)-K$.

When the option has an infinite maturity, the valuation formulas have especially simple forms. Equations (2) and (3) square with intuition in several senses. They show that the value of the infinite option is an increasing function of the cap, a decreasing function of the strike price, and is bounded above by the exercise payoff $L-K$ and below by zero.

Valuation formulas for finite-maturity capped options are given next. Let $\tau_{L}=\inf \left\{\nu \in[t, \infty): S_{\nu} \geq L\right\}$ represent the first hitting time of the set $[L, \infty)$, that is, the first time at which the value of the underlying dividend paying asset equals or exceeds $L$. Let $t^{*}$ be defined by the solution to the equation $B_{v}=L_{v}$ for $v \in[0, \mathrm{~T}]$ if a solution exists. ${ }^{5}$ If $B_{v}<L$ for all $\boldsymbol{v} \in[0, T]$ set $t^{*}=0$, and if $B_{v}>L$ for all $\boldsymbol{v} \in[0, \mathrm{~T}]$ set $t^{*}=T$. Also, let $C_{t}\left(S_{t}\right)$ denote the value of an American uncapped call option with maturity $T$ on the same dividend-paying underlying asset. Throughout the article, $n(z)$ represents the density function of a standard normal random variable and $N(z)$ denotes the cumulative distribution function of a standard normal random variable.

Theorem 2. Consider an American call option with exercise price $K$, cap equal to $L$, and maturity $T$. For $S_{t} \geq L \wedge B_{t}$ the option value is $\left(S_{t} \wedge L\right)-K$. For $S_{t}<L \wedge B_{t}$ and $t \geq t^{*}$, the option value is $C_{t}\left(S_{t}\right)$. For $S_{t}<L \wedge B_{t}$ and $t<t^{*}$, the option value is given by

$$
\begin{aligned}
C_{t}^{L}\left(S_{t}, K, T-t\right)= & E_{t}^{*}\left[e^{-r\left(\tau_{L}-t\right)}\left(S_{\tau_{L}}-K\right) 1_{\left\{\tau_{L}<r\right\}}\right] \\
& +E_{t}^{*}\left[e^{-r\left(r^{*}-t\right)} C_{r}\left(S_{t}\right) 1_{\left\{\tau_{L} \geq r\right]}\right]
\end{aligned}
$$

The valuation formula for $C_{\boldsymbol{t}}^{\boldsymbol{L}}\left(\boldsymbol{S}_{\mathfrak{l}}, \boldsymbol{K}, \boldsymbol{T}-\boldsymbol{t}\right)$ can be written more explicitly as

$$
\begin{gathered}
(L-K)\left[\lambda_{t}^{2 \phi / \sigma^{2}} N\left(d_{0}\right)+\lambda_{t}^{2 \alpha / \sigma^{2}} N\left(d_{0}+2 f \sqrt{t^{*}-t} / \sigma\right)\right] \\
+e^{-r(r-t)} \int_{x=0}^{L} C_{t}(x) u\left(x, t, t^{*}\right) d x
\end{gathered}
$$

\footnotetext{
${ }^{4}$ For $S_{t}<\mathrm{L}$, equation (3) in the absence of dividends is valid for more general price proceses. In fact, the last part of the proof of Corollary 1 in the Appendix shows that the equation holds for Itô price processes $\boldsymbol{d} \boldsymbol{S}_{\boldsymbol{l}}=\boldsymbol{S}_{\boldsymbol{t}}\left[\boldsymbol{r} \boldsymbol{d} \boldsymbol{t}+\boldsymbol{\sigma}_{\boldsymbol{l}} \boldsymbol{d} \boldsymbol{W}_{\boldsymbol{l}}\right]$, ' where $r$ is constant and $\sigma_{\mathrm{t}}$ is progressively measurable with respect to $W$. We thank Bjorn Flesaker for pointing out the generality of equation (3).

${ }^{5}$ Since $L$ is constant and $B_{t}$ is continuous and strictly decreasing in $t$, there cannot be more than one solution $t^{*}$.
} 
where

$$
\begin{aligned}
u\left(x, t, t^{*}\right) & =\frac{1}{x \sigma \sqrt{t^{*}-t}}\left[n\left(d_{1}^{-}(x)\right)-\lambda_{t}^{1-2(r-\delta) / \sigma^{2}} n\left(d_{1}^{+}(x)\right)\right], \\
d_{0} & =\frac{1}{\sigma \sqrt{t^{*}-t}}\left[\log \left(\lambda_{t}\right)-f\left(t^{*}-t\right)\right], \\
d_{1}^{ \pm}(x) & =\frac{1}{\sigma \sqrt{t^{*}-t}}\left[ \pm \log \left(\lambda_{t}\right)-\log (L)+\log (x)+b\left(t^{*}-t\right)\right], \\
b & =\delta-r+\frac{1}{2} \sigma^{2}, \quad f=\sqrt{b^{2}+2 r \sigma^{2}}, \\
\phi & =\frac{1}{2}(b-f), \quad \alpha=\frac{1}{2}(b+f), \text { and } \lambda_{t}=S_{t} / L .
\end{aligned}
$$

In Theorem 2 and elsewhere in the article, $\boldsymbol{E}_{\boldsymbol{t}}^{*}$ denotes the expectation operator with respect to the equivalent (risk-neutral) martingale measure and the subscript $t$ denotes conditioning on the information at date $t$. The risk-neutral representation formula in equation (4) is standard [see, for instance, Harrison and Kreps (1979)].

When the dividend rate is sufficiently small the optimal exercise boundary for the uncapped option lies above the cap. In particular, when $\delta \leq r K / L, B_{v}$ f o r a $1 l v \in[0, T]$.In this case, $t^{*}=T$, the optimal exercise policy is to exercise at the cap, and equation (5) can be written more explicitly. The resulting expression for the option value is given next in Corollary 2. Equation (10) was stated in Rubinstein and Reiner (1991) for the value of a capped option with automatic exercise at the cap.

Corollary 2 (American capped call valuation with low dividends). Suppose that the underlying asset's price follows the geometric Brownian motion process specified in equation (1). Also, suppose that $\boldsymbol{\delta} \leq \boldsymbol{r} K / L$. Then the value of an American capped call option, for $S_{t} \leq L$ and $t \in[0, T]$, is

$$
\begin{aligned}
C_{t}^{L}\left(S_{t}, K, T-t\right) & \\
= & (L-K)\left[\lambda_{t}^{2 \phi / \sigma^{2}} N\left(d_{0}\right)+\lambda_{t}^{2 \alpha / \sigma^{2}} N\left(d_{0}+2 f \sqrt{T-t} / \sigma\right)\right] \\
& +S_{t} e^{-\delta(T-t)}\left[N\left(d_{1}^{-}(L)-\sigma \sqrt{T-t}\right)\right. \\
& \left.-N\left(d_{1}^{T}(K)-\sigma \sqrt{T-t}\right)\right] \\
& -\lambda_{t}^{-2(r-\delta) / \sigma^{2}} L e^{-\delta(T-t)}\left[N\left(d_{1}^{+}(L)-\sigma \sqrt{T-t}\right)\right. \\
& \left.-N\left(d_{1}^{+}(K)-\sigma \sqrt{T-t}\right)\right]
\end{aligned}
$$




$$
\begin{aligned}
& -K e^{-r(T-t)}\left[N\left(d_{1}^{-}(L)\right)-N\left(d_{1}^{-}(K)\right)\right. \\
& \left.-\lambda_{t}^{1-2(r-\delta) / \sigma^{2}}\left[N\left(d_{1}^{+}(L)\right)-N\left(d_{1}^{+}(K)\right)\right]\right] .
\end{aligned}
$$

In (10) the expressions for $\boldsymbol{d}_{0}$ and $\boldsymbol{d}_{1}^{ \pm}(\boldsymbol{x})$ are the same as in (7) and (8) but with $T-t$ replacing $t^{*}-t$. The expressions for $b, f, \phi, \alpha$, and $\lambda_{t}$ are the same as in (9).

Corollary 3 (European capped call valuation). Let $C_{t}^{E}\left(S_{t}, L, K\right.$, $T-t)$ represent the value of an option at time $t$ that has a strike price of $K$, a cap of $L$, a maturity of $T$, and which cannot be exercised until maturity. Then the value of this European capped call is given by

$$
\begin{aligned}
& C_{t}^{E}\left(S_{t}, L, K, T-t\right) \\
&= S_{t} e^{-\delta(T-t)}\left[N\left(d_{1}^{-}(L)-\sigma \sqrt{T-t}\right)-N\left(d_{1}^{-}(K)-\sigma \sqrt{T-t}\right)\right] \\
&-K e^{-r(T-t)}\left[1-N\left(d_{1}^{-}(K)\right)\right]+L e^{-r(T-t)}\left[1-N\left(d_{1}^{-}(L)\right)\right] .
\end{aligned}
$$

The expression for $\boldsymbol{d}_{1}^{-}(\boldsymbol{x})$ is the same as in (8) but with $T$ - $t$ replacing $t *-t$.

Since the European capped call does not allow for early exercise, its price is a lower bound on the price of the American capped call option. That is, $C_{t}^{E} \leq C_{t}^{\boldsymbol{L}}$. If $\boldsymbol{L} \uparrow \infty$ in equation (11) then

$$
\begin{aligned}
C_{t}^{E}\left(S_{t}, \infty, K, T-t\right)= & S_{t} e^{-\delta(T-t)}\left[1-N\left(d_{1}^{-}(K)-\sigma \sqrt{T-t}\right)\right] \\
& -K e^{-r(T-t)}\left[1-N\left(d_{1}(K)\right)\right],
\end{aligned}
$$

which is the Black-Scholes European option formula adjusted for dividends [Black and Scholes (1973)].

\section{American Capped Calls with Delayed Exercise Periods}

Some American capped call options, such as the MILES contract, involve restrictions on the time period in which exercise is allowed. If the underlying stock price follows a geometric Brownian motion process, Theorem 2 can be generalized to handle the delayed exercise period.

Theorem 3 (American capped call with delayed exercise period). Suppose that the underlying asset's price follows a geometric Brownian motion process and consider an American capped call 
option with exercise period equal to $\left[t_{e}, T\right]$. The value at time $t \leq t_{e}$ is

$$
\begin{aligned}
& C_{t}^{L}\left(S_{t}, T-t, t_{e}\right) \\
& =(L-K) e^{-r\left(t_{t}-t\right)} E_{t}^{*}\left[1_{\left(S_{t} \geq L\right\}}\right]+e^{-r\left(t_{t}-t\right)} E_{t}^{*}\left[C_{t_{0}}^{L}\left(S_{t_{0}}, T-t_{e}\right) 1_{\left\{S_{b}<L\right\}}\right] \\
& =(L-K) e^{-r\left(t_{0}-t\right)}\left[1-N\left(d_{1}\left(L, t_{e}-t\right)\right)\right] \text {. } \\
& +e^{-r\left(t_{e}-t\right)} \int_{z=-\infty}^{\alpha_{1}\left(L, t_{t}-t\right)} C_{t_{s}}^{L}\left(S_{t} e^{\left(r-\delta-\frac{1}{2} \sigma^{2}\right)\left(t_{t}-t\right)+\sigma z \sqrt{t_{t}-t}}, T-t_{e}\right) n(z) d z .
\end{aligned}
$$

In (13), $C_{\mathfrak{L}}^{L}\left(S_{\boldsymbol{h}}, T-\boldsymbol{t}_{\boldsymbol{e}}\right)$ denotes the value of the American capped call option from Theorem 2 with $T-t_{e}$ replacing $T-t$ and $\max \left(t^{*}, t_{e}\right)$ replacing $t^{*}$ in equation (5), and where $\boldsymbol{d}_{\mathbf{1}}^{-}\left(\boldsymbol{x}, \boldsymbol{t}_{\boldsymbol{e}}-\boldsymbol{t}\right)$ is given in (8) with $t_{e}-t$ replacing $t^{*}-t$.

The optimal exercise policy is to exercise at time $t_{e}$ if $\boldsymbol{S}_{\boldsymbol{b}} \geq \boldsymbol{L} \wedge \boldsymbol{B}_{\boldsymbol{t}_{e}}$. Otherwise, exercise at the first time after $t_{e}$ that $S_{t}$ reaches $L \wedge B_{t}$, and if this does not occur then exercise at time $T$. The formula given in (13) simplifies when the maturity of the contract is infinite.

\subsection{Capped call options on the dollar value of an index}

The MILES security is an American capped call option on the dollar value of the Mexican stock index with a delayed exercise period. The previous analysis is applied to this type of security next.

Let $S_{t}=e_{t} M_{t}$ represent the dollar value of the Mexican stock index, where $e_{t}$ is the dollar-peso (\$/peso) exchange rate and $M_{t}$ is the value in pesos of the Mexican stock index (all at time $t$ ). Suppose that $e_{t}$ and $M_{t}$ follow the geometric Brownian motion processes

$$
\begin{aligned}
& d e_{t}=e_{t}\left[\mu_{e} d t+\sigma_{a_{1}} d W_{1 t}+\sigma_{e_{2}} d W_{2 t}\right] \\
& d M_{t}=M_{t}\left[\left(\mu_{M}-\delta_{M}\right) d t+\sigma_{M_{1}} d W_{1 t}+\sigma_{M_{2}} d W_{2 i}\right]
\end{aligned}
$$

where the volatility coefficients $\boldsymbol{\sigma}_{\boldsymbol{Q}_{1}}, \boldsymbol{\sigma}_{\boldsymbol{Q}_{2}}, \boldsymbol{\sigma}_{\boldsymbol{M}_{1}}$, and $\boldsymbol{\sigma}_{\boldsymbol{M}_{2}}$ are constant, the drifts $\mu_{e}$ and $\mu_{M}$ are constant, $\delta_{M}$ is the constant dividend rate of the. Mexican index, and $W_{1 t}$ and $W_{2 t}$ are independent Brownian motion processes.

With these assumptions, Itô's lemma implies that the dollar value of the Mexican index satisfies

$$
d S_{t}=S_{t}\left[\left(\mu_{e}+\mu_{M}+c\right) d t+\left(\sigma_{Q_{1}}+\sigma_{M_{1}}\right) d W_{1 t}+\left(\sigma_{e_{2}}+\sigma_{M_{2}}\right) d W_{2 t}\right]
$$

where: $c=\sigma_{\mathrm{A}_{1}} \sigma_{M_{1}}+\sigma_{e_{2}} \sigma_{M_{2}}$ is the (local) correlation between the processes $e$ and $M$. 
Since the volatility coefficients are assumed to be constant, the only risk which matters is

$$
\begin{aligned}
d W_{t} \equiv & \frac{1}{\sqrt{\left(\sigma_{Q_{1}}+\sigma_{M_{1}}\right)^{2}+\left(\sigma_{e_{2}}+\sigma_{M_{2}}\right)^{2}}} \\
& \times\left[\left(\sigma_{Q_{1}}+\sigma_{M_{1}}\right) d W_{1 t}+\left(\sigma_{Q_{2}}+\sigma_{M_{2}}\right) d W_{2 t}\right]
\end{aligned}
$$

which is a Brownian motion process. This risk can be hedged away by trading in the dollar value of the index. Hence formula (13) of Theorem 3 applies with $\sigma=\sqrt{\left(\sigma_{Q_{1}}+\sigma_{M_{1}}\right)^{2}+\left(\sigma_{Q_{2}}+\sigma_{M_{2}}\right)^{2}}$.

\section{Caps with a Constant Growth Rate}

The MILES option contract has a delayed exercise period which can be viewed as a time-varying cap. In this case, the cap is a step function with a single jump. Options with time-varying caps - more specifically, growing caps - may be preferred by investors over constant caps. Increasing caps enable investors to capture more upside potential, and so may increase the attractiveness of the contract from their point of view. Also, issuers may be prepared to accept an increasing potential liability as time passes in return for a higher premium today. In this section we analyze time-varying caps that grow at a constant rate $g \geq 0 .^{6}$ That is, the cap as a function of time is

$$
L_{t}=L_{0} e^{g t} \text {, }
$$

\&here we assume $L_{0}>K$.

For time-varying caps given by equation (14), the optimal exercise policy depends on three parameters. To define the policy, recall that $B_{t}$ denotes the optimal exercise boundary for an uncapped call option with the same maturity and strike price as the capped call option. Let $t^{*}$ be defined by the solution to the equation $B_{v}=L_{v}$ for $v \in[0, \mathrm{~T}]$, if a solution exists. If $B_{v}<L_{v}$ for all $\boldsymbol{v} \in[0, T]$, set $\boldsymbol{t}^{*}=0$, and if $\boldsymbol{B}_{\boldsymbol{v}}>\boldsymbol{L}_{\boldsymbol{v}}$ for all $v \in[t, T]$, set $t^{*}=T$.

Definition $1 \quad\left(\left(t_{e}, \boldsymbol{r}, t_{f}\right)\right.$ Exercise Policy). Let $t_{e}$ and $t_{f}$ satisfy $0 \leq$ $t_{e} \leq t_{f} \leq T$ and $t_{e} \leq t^{*} \leq T$. Define the stopping time $\tau_{1} b y$ inf $\{v \in$ $\left.\left\{\boldsymbol{t}_{\boldsymbol{e}}, \boldsymbol{t}_{\boldsymbol{f}}\right]: \boldsymbol{S}_{\boldsymbol{v}}=\boldsymbol{L}_{\boldsymbol{v}}\right\}$ or, if no such $v$ exists, set $\boldsymbol{\tau}_{\mathbf{1}}=T$. Set the stopping time $\boldsymbol{\tau}_{\mathbf{2}}$ equal to $t_{f}$ if $S_{t f} \geq L_{t f}$; otherwise set $\boldsymbol{\tau}_{\mathbf{2}}=T$. Define the stopping time $\boldsymbol{\tau}_{\mathbf{3}}$ byinf $\left\{\boldsymbol{v} \in\left[\boldsymbol{t}^{*}, \boldsymbol{T}\right]: \boldsymbol{S}_{\boldsymbol{v}}=\boldsymbol{B}_{\boldsymbol{v}}\right\}$ or, if no such $v$ exists, set $\boldsymbol{\tau}_{\mathbf{3}}=T$. An exercise policy is $a\left(t_{e}, t^{*}, t_{f}\right)$ policy if the option is exercised at the stopping time $\min \left\{\boldsymbol{\tau}_{\mathbf{1}}, \boldsymbol{\tau}_{\mathbf{2}}, \boldsymbol{\tau}_{\mathbf{3}}\right\}$.

${ }^{6}$ More general results for time-varying and random caps in the absence of dividends are given in Broadie and Detemple (1993). 


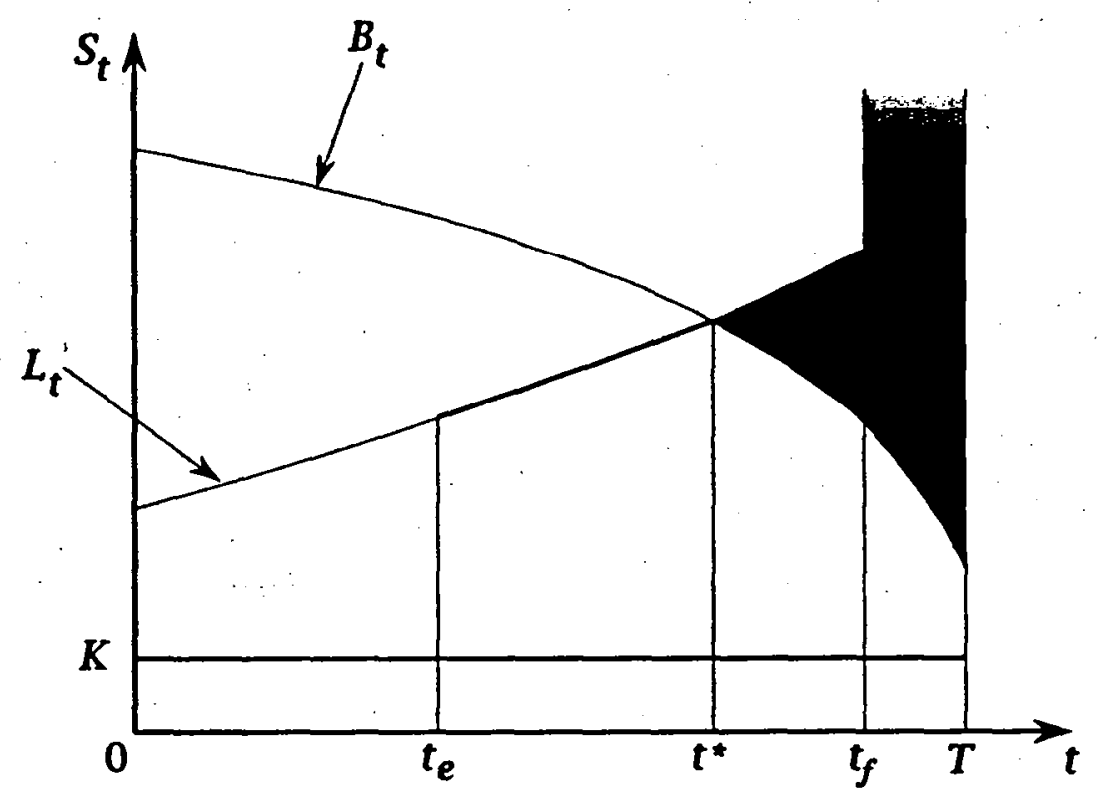

Figure 2

Exercise region for a $\left(t_{e}, t^{*}, t_{f}\right)$ policy

A typical exercise region corresponding to a $\left(t_{e}, t^{*}, t_{f}\right)$ exercise policy is illustrated in Figure 2. The exercise region is indicated by the shaded area together with the darker line segment joining $L_{t e}$ to $L_{t^{*}}$. If a $\left(t_{e}, t^{*}, t_{f}\right)$ policy is optimal, then it is not optimal to exercise prior to $t_{e}$ even if the underlying asset's price equals the cap. Prior to $t_{f}$ it is not optimal to exercise if the underlying asset's price is strictly above the cap. The shaded region from $t_{f}$ to $T$ collapses to a vertical line when $t_{f}=T$. In the case of a constant cap, $t_{e}=t_{f}=0$, and the optimal policy simplifies to the one described in Theorem 1.

The main results are given next. The optimal exercise policy is characterized in Theorem 4. A formula for valuing a capped call option for any $\left(t_{e}, t^{*}, t_{f}\right)$ policy is given in Theorem 5. Finally, equations characterizing the optimal values of $t_{e}$ and $t_{f}$ are given in Theorem 6 .

Theorem 4. Consider an American call option with exercise price $K$, cap given by equation (14), and maturity $T$. Then the optimal exercise policy is $a\left(t_{e}, t^{*}, t_{f}\right)$ policy.

In the absence of dividends, $B_{t}=\infty$ for all $t$ and $t^{*}=T$. In this case, Theorem 4 shows that the optimal exercise policy reduces to a twoparameter $\left(t_{e}, t_{f}\right)$ policy where exercise below the cap is not optimal. 
Some intuition behind Theorem 4 follows. It is shown in the proof of Theorem 6 that the present value at time $t$ of $L_{v}-K$ is strictly increasing with respect to $v$ up to some $t_{f}^{*}$ [defined in equation (18) below], and strictly decreasing thereafter. Hence, a waiting strategy is optimal if $S_{t}>L_{t}$ and $t<t_{f}^{*}$, while immediate exercise is optimal if $S_{t} \geq L_{t}$ and $t \geq t_{f}^{*}$. If $t^{*} \leq t \leq T$ and $B_{t} \leq S_{t} \leq L_{t}$, then immediate exercise is optimal. This follows since immediate exercise is optimal for an uncapped option and since the holder of the capped option can attain (but cannot improve upon) this value by exercising immediately. If $S_{t}<L_{t} \wedge B_{t}$ then immediate exercise is suboptimal by an argument similar to the one in the proof of Theorem 1 .

Theorem 5 gives a valuation formula for a capped call option for a given $\left(t_{e}, t^{*}, t_{f}\right)$ exercise policy.

Theorem 5. Consider American call option with exercise price $K$, cap given by equation (14), and maturity T. Suppose that a $\left(t_{e}, t^{*}, t_{f}\right)$ exercise policy is followed for some fixed values of $t_{e}$ and $t_{f}$. The value at time $t \leq t_{e}$ corresponding to this policy is given by

$$
\begin{aligned}
& C_{t}^{L}\left(t_{e}, t^{*}, t_{f}\right)
\end{aligned}
$$

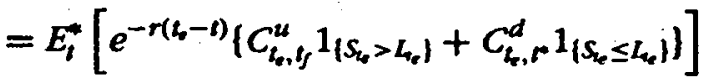

$$
\begin{aligned}
& =e^{-r\left(t_{0}-t\right)} \int_{z=-\bar{d}_{2}^{+}\left(\lambda_{1}, t_{0}-t\right)}^{\infty} C_{t_{0}, t_{f}}^{u}\left(L_{t_{f}}, \lambda_{t} e^{-\bar{b}\left(t_{t}-t\right)+\sigma z \sqrt{t_{0}-t}}, t_{f}-t_{e}\right) n(z) d z
\end{aligned}
$$

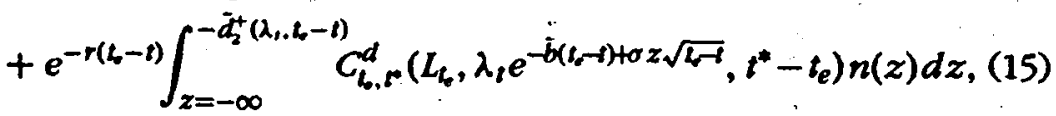

where $\lambda_{t}=S_{t} / L_{t}, \bar{b}=g+\delta-r+\frac{1}{2} \sigma^{2}, \bar{d}_{2}^{+}\left(\lambda_{t}, t_{e}-t\right)=\emptyset \log \left(\lambda_{t}\right)-\bar{b}\left(t_{e}-\right.$ $t)] /\left(\sigma \sqrt{t_{e}-t}\right)$, and $C_{t_{b}, t}^{u}$, and $C_{t, r}^{d}$ are defined below.

At time $t_{e}$ with $S_{t e}>L_{t e}$, the value corresponding to the $\left(t_{e}, t^{*}, t_{f}\right)$ policy is independent of $t^{*}$ and is given by $C_{t_{*}, t_{f}}^{\boldsymbol{u}}$ iwhere

$$
\begin{aligned}
& C_{t_{0} t_{f}}^{u}\left(L_{t_{s}}, \lambda_{t_{s}}, t_{f}-t_{e}\right) \\
& =L_{t}\left[\lambda_{t_{b}}^{2 \bar{\phi} / \sigma^{2}} N\left(-\bar{d}_{0}\right)+\lambda_{t_{b}}^{2 \bar{\alpha} / \sigma^{2}} N\left(-\bar{d}_{0}-2 \bar{f} \sqrt{\left(t_{f}-t_{e}\right)} / \sigma\right)\right] \\
& -K\left[\lambda_{t_{o}}^{2 \phi / \sigma^{2}} N\left(-d_{0}\right)+\lambda_{t_{t}}^{2 \alpha / \sigma^{2}} N\left(-d_{0}-2 f \sqrt{\left(t_{f}-t_{e}\right) / \sigma}\right)\right] \\
& +\left[L_{t_{e}} e^{g\left(t_{f}-t_{6}\right)}-K\right] e^{-r\left(t_{f}-t_{0}\right)}\left[N\left(d_{2}^{+}\right)-\lambda_{l_{b}}^{1+2(g+\delta-r) / \sigma^{2}} N\left(d_{2}^{-}\right)\right],(16)
\end{aligned}
$$

and wbere $\lambda_{t_{6}}=S_{t_{b}} / L_{t_{b}}, \bar{b}=g+\delta-r+\frac{1}{2} \sigma^{2}, d_{0}=\left[\log \left(\lambda_{i_{0}}\right)-f\left(t_{f}-\right.\right.$ $\left.\left.t_{e}\right)\right] /\left(\sigma \sqrt{t_{f}-t_{e}}\right), \bar{d}_{0}=\left[\log \left(\lambda_{t_{s}}\right)-\bar{f}\left(t_{f}-t_{e}\right)\right] /\left(\sigma \sqrt{t_{f}-t_{e}}\right), f=\left(\bar{b}^{2}+\right.$ $\left.2 \sigma^{2} r\right)^{1 / 2}, \bar{f}=\left(\bar{b}^{2}+2 \sigma^{2}(r-g)\right)^{1 / 2}, \phi=\frac{1}{2}(\bar{b}-f), \bar{\phi}=\frac{1}{2}(\bar{b}-\bar{f}), \alpha=$ 
$\frac{1}{2}(\bar{b}+f) ; \bar{\alpha}=\frac{1}{2}(\bar{b}+\bar{f})$, and $d_{2}^{ \pm}=\left[ \pm \log \left(\lambda_{t_{o}}\right)-\bar{b}\left(t_{f}-t_{e}\right)\right] /\left(\sigma \sqrt{t_{f}-t_{e}}\right)^{7}$

At time $t_{e}$ with $S_{t e} \leq L_{t}$, the value corresponding to the $\left(t_{e}, t^{*}, t_{f}\right)$ policy is given by $C_{t_{0}, \text { w }}^{d}$ where

$$
\begin{aligned}
& C_{t_{e}, F}^{d}\left(L_{t_{e}}, \lambda_{b_{b}}, t^{*}-t_{e}\right) \\
& =L_{t_{*}}\left[\lambda_{t_{t}}^{2 \bar{\phi} / \sigma^{2}} N\left(\bar{d}_{0}\right)+\lambda_{t_{e}}^{2 \bar{\alpha} / \sigma^{2}} N\left(\bar{d}_{0}+2 \bar{f} \sqrt{\left(t^{*}-t_{e}\right)} / \sigma\right)\right] \\
& -K\left[\lambda_{t_{e}}^{2 \phi / \sigma^{2}} N\left(d_{0}\right)+\lambda_{t_{6}}^{2 \alpha / \sigma^{2}} N\left(d_{0}+2 f \sqrt{\left(t^{*}-t_{e}\right)} / \sigma\right)\right] \\
& +\int_{x=0}^{L_{*}} e^{-r\left(t^{*}-t_{*}\right)} C_{t}(x) \bar{u}\left(x, t_{e}, t^{*}\right) d x \text {, }
\end{aligned}
$$

and where $\bar{u}\left(x, t_{e}, t^{*}\right)=\left[n\left(\bar{d}_{1}(x)\right)-\lambda_{h_{e}}^{1-2(r-\delta-g) / \sigma^{2}} n\left(\bar{d}_{1}^{+}(x)\right)\right] /$ $\left(x \sigma \sqrt{t^{*}-t_{e}}\right), \bar{d}_{1}^{ \pm}(x)=\left[ \pm \log \left(\lambda_{L_{b}}\right)-\log \left(L_{t_{e}}\right)+\log (x)+(\bar{b}-g)\left(t^{*}-\right.\right.$ $\left.\left.\left.t_{e}\right)\right] /\left(\sigma \sqrt{t^{*}-t_{e}}\right)\right] /\left(\sigma \sqrt{t^{*}-t_{e}}\right), d_{0}=\left[\log \left(\lambda_{k_{e}}\right)-f\left(t^{*}-t_{e}\right)\right] /\left(\sigma \sqrt{t^{*}-t_{e}}\right)$, and $\bar{d}_{0}=\left[\log \left(\lambda_{t_{e}}\right)-\bar{f}\left(t^{*}-t_{e}\right)\right] /\left(\sigma \sqrt{t^{*}-t_{e}}\right)$.

The value at time $t \leq t_{e}$ under the $\left(t_{e}, t^{*}, t_{f}\right)$ policy is the present value of the corresponding policy at time $t_{e}$. This present value is the risk-neutral probability weighted average of the possible values on the event $\left\{S_{t e}>L_{t e}\right\}$ and the event $\left\{S_{t e} \leq L_{t e}\right\}$. This observation leads to equation (15).

Theorem 6 characterizes the optimal values of $t_{e}$ and $t_{f}$ in a $\left(t_{e}, t^{*}, t_{f}\right)$ exercise policy.

Theorem 6. Consider an American call option with exercise price $K$, cap given by equation (14), and maturity T. Let $t_{f}^{*}$ denote the optimal due of $t_{f}$ in $a\left(t_{e}, t^{*}, t_{f}\right)$ policy. Then $t_{f}^{*}$ is given by

$$
t_{f}^{*}=\underset{0 \leq s \leq T}{\operatorname{argmax}} e^{-r s}\left[L_{0} e^{g s}-K\right]
$$

In particular, if

$$
(g-r) L_{0} e^{g T}+r K \geq 0,
$$

then $\boldsymbol{t}_{f}^{*}=T$. Also, if

$$
(g-r) L_{0}+r K \leq 0,
$$

then $\boldsymbol{t}_{\boldsymbol{f}}^{*}=0$. Otherwise, $0<\boldsymbol{t}_{\boldsymbol{f}}^{*}<T$ and $\boldsymbol{t}_{\boldsymbol{f}}^{*}$ is given by

$$
t_{f}^{*}=\frac{1}{g} \log \left(\frac{r K}{(r-g) L_{0}}\right)
$$

Note that $\overline{\boldsymbol{b}}$,differs from $\mathrm{b}$ because of $\mathrm{g}$. Also $f, \phi$, and $\alpha$ have slightly different meanings than before because of $\bar{b}$ in their definitions. 
Let $t_{e}^{*}$ denote the optimal value of $t_{e}$ in $a\left(t_{e}, t^{*}, t_{f}^{*}\right)$ exercise policy. The following conditions are satisfied by $\boldsymbol{t}_{\boldsymbol{e}}^{*}$. First, if $0<\boldsymbol{t}_{\boldsymbol{e}}^{*}<\boldsymbol{t}^{*} \wedge \boldsymbol{t}_{\boldsymbol{f}}^{*}$, then $t_{e}{ }^{*}$ is a solution to equation (22):

$$
\begin{aligned}
& \int_{\frac{b}{\sigma} \sqrt{t}}^{\infty}\left[-r C_{t_{0}, f_{f}}^{u}+g L_{t_{e}} \frac{\partial C_{t_{s}, r_{f}}^{u}}{\partial L_{t_{b}}}\right. \\
& \left.-\left(\bar{b}-\frac{1}{2} \frac{\sigma z}{\sqrt{t_{e}}}\right) \lambda_{t_{e}} \frac{\partial C_{t_{e}, f_{f}}^{u}}{\partial \lambda_{t_{e}}}-\frac{\partial C_{t_{0}, r_{f}}^{u}}{\partial\left(t_{f}^{*}-t_{e}\right)}\right] n(z) d z \\
& +\int_{-\infty}^{\frac{b}{6} \sqrt{6}}\left[-r C_{t_{0}, 5}^{d}+g L_{t_{0}} \frac{\partial C_{t_{c}, F}^{d}}{\partial L_{t_{t}}}\right. \\
& \left.-\left(\bar{b}-\frac{1}{2} \frac{\sigma z}{\sqrt{t_{e}}}\right) \lambda_{t_{e}} \frac{\partial C_{t_{e}, \Gamma}^{d}}{\partial \lambda_{t_{e}}}-\frac{\partial C_{t_{e}, \Gamma}^{d}}{\partial\left(t^{*}-t_{e}\right)}\right] n(z) d z=0 \text { (22) }
\end{aligned}
$$

Second, if $\boldsymbol{t}_{\boldsymbol{e}}^{*}=\boldsymbol{t}^{*} \wedge \boldsymbol{t}_{f}^{*}$ then the lefthand side of (22) is nonnegative. Third, if $t^{*}{ }_{e}=0$, the lefthand side of equation (22) has a nonpositive limit as $t_{e} \downarrow 0 .{ }^{8}$

The value of the American option at time $t \leq t^{*}{ }_{e}$ is then given by equation (15) evaluated at $t_{e}=t^{*}{ }_{e}$ and $t_{f}=t^{*}{ }_{f}$

Theorem 6 specifies a three-step procedure for valuing American capped call options where the cap has a constant growth rate. First, $t^{*}{ }_{f}$ is found, then $t^{*}{ }_{e}$ is found, and finally the option value is determined. The optimal value $t_{f}^{*}$ solves (18). Note that if $g \geq r$ then condition (19) holds and $t_{f}^{*}=T$, The optimal $t^{*}{ }_{e}$ is the time that maximizes the option value given in equation (15) when $t_{f}=t^{*}{ }_{f}$ For interior solutions, that is, when $0<t^{*}{ }_{e}<t^{*} \wedge t_{f}^{*}$, the optimal $t^{*}{ }_{e}$ solves $\partial C_{0}^{L}\left(t_{e}, t^{*}, t_{f}^{*}\right) /\left.\partial t_{e}\right|_{\boldsymbol{t}_{0}=t_{\boldsymbol{c}}}=0$. This optimal time balances the marginal benefit (or loss) of increasing $t_{e}$ on the event $\left\{\mathrm{S}_{\mathrm{te}}>\mathrm{L}_{\mathrm{te}}\right\}$ with the marginal loss (or benefit) on the event $\left\{\mathrm{S}_{\mathrm{te}} \leq \mathrm{L}_{\mathrm{te}}\right\}$. For boundary solutions, that is, when, $t_{e}^{*}=0$ or $t_{e}^{*}=t^{*} \wedge t_{f}^{*}$, the equality of the marginal benefit to the marginal loss is replaced by the appropriate inequality. Once $t_{e}^{*}$ and $t_{f}^{*}$ are determined, the option value is given by equation (15).

Equation (21) reveals how $t_{f}^{*}$ depends on the parameters $r, g, K$, and $L_{0}$. First, the optimal time $\boldsymbol{t}_{\boldsymbol{f}}^{*}$ is independent of $\sigma$ and $\delta$. Also, a

Explicit expressions for all of the partial derivatives in equation (22) can be obtained from the authors. 
decrease in the riskless rate $r$ or an increase in the ratio $K / L_{0}$ will increase $\boldsymbol{t}_{\boldsymbol{f}}^{*}$.An increase in the rate of growth $g$ increases $\boldsymbol{t}_{\boldsymbol{f}}^{*}$ if and only if $\boldsymbol{t}_{f}^{*}<g /(r-g)$.

Numerical results are given in the next section. These results indicate that the magnitude of the loss when following the policy of exercising at the cap instead of the optimal $\left(\boldsymbol{t}_{\boldsymbol{e}}^{*}, \boldsymbol{t}^{*}, \boldsymbol{t}_{\boldsymbol{f}}^{*}\right)$ policy can be substantial. Additional results illustrate how $t^{*}{ }_{e}$ varies with $g$ and $\sigma$.

\section{Computational Results}

In this section we provide computational results using the valuation formulas derived in this article. The results are used to qualitatively compare the behavior of capped versus uncapped and American versus European option values.

In the presence of dividends, the valuation formulas (5), (13), and (17) can be difficult to evaluate. Broadie and Detemple (1994) give a fast way to approximate the exercise boundary which appears in (5), (13), and (17). Boyle and Law (1994) discuss effective modifications of the Cox, Ross, and Rubinstein (1979) binomial procedure for the valuation of capped options. Here, to simplify the presentation, we consider the case of no dividends (that is, $\delta=0$ ) throughout this section.

\subsection{Comparison of option prices and the early exercise premium}

Figure 3 shows a comparison between: (i) standard European uncapped call option, (ii) American capped call option, (iii) American capped call option with delayed exercise period, and (iv) European capped call option. The option values are computed from equations (12), (10), (13), and (11) for options (i)-(iv), respectively. The parameters for the comparison are $r=0.05, \sigma=0.2, T=1$, $t=0, K=30, L=60$, and $t_{e}=0.5$ [for option (iii)]. The option values are plotted versus $S_{0}$, where $S_{0}$ ranges from 35 to 75 .

Since option values are increasing functions of the cap, it must be that the option values satisfy (i) $\geq$ (ii) $\geq$ (iii) $\geq$ (iv). This ordering of option values is illustrated in Figure 3. For stock values well below the cap, that is, for $S_{0} \ll L$, the four option values are nearly identical. This makes sense, since the probability of the stock price exceeding the cap by the maturity date approaches zero as $S_{0} \downarrow 0$.

The early exercise premium is the difference between the American and European option values. For uncapped call options on nondividend paying assets the early exercise premium is zero, that is, early exercise is not optimal. For capped call options, the early exercise 


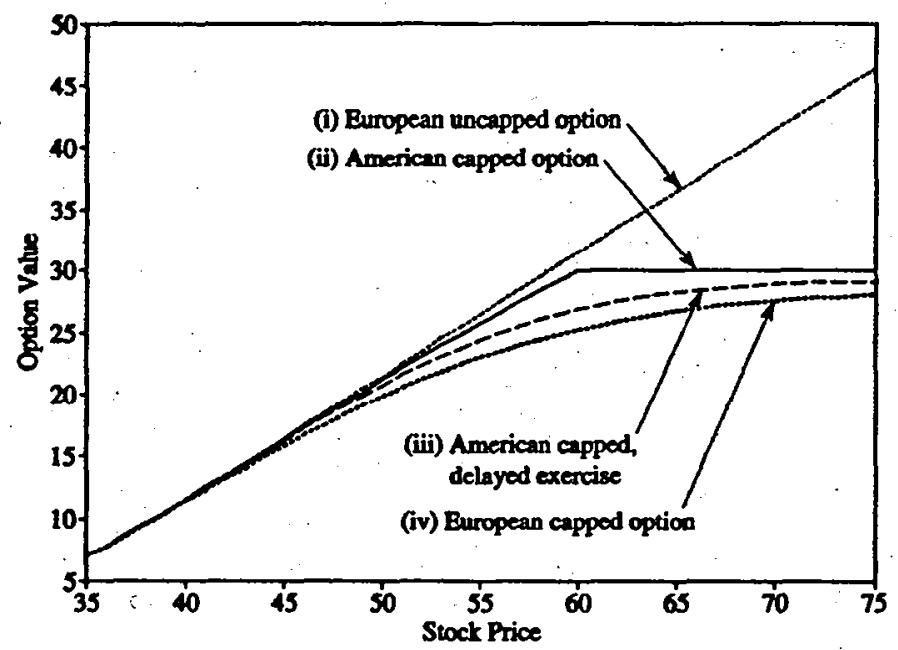

Figure 3

Comparison of option values for different stock prices

Table 1

Early exercise premium

\begin{tabular}{lcccc}
\hline $\begin{array}{l}\text { Stock } \\
\text { price }\end{array}$ & $\begin{array}{c}\text { European capped } \\
\text { option value }(a)\end{array}$ & $\begin{array}{c}\text { American capped } \\
\text { option value }(b)\end{array}$ & $\begin{array}{c}\text { Early exercise } \\
\text { premium }(c=b-a)\end{array}$ & $c / a(\%)$ \\
\hline 35 & 6.95 & 6.96 & 0.02 & 0.27 \\
40 & 11.45 & 11.57 & 0.12 & 1.08 \\
45 & 15.91 & 16.40 & $\mathbf{0 . 4 9}$ & $\mathbf{3 . 0 5}$ \\
$\mathbf{5 0}$ & 19.85 & 21.17 & $\mathbf{1 . 3 2}$ & $\mathbf{6 . 6 6}$ \\
$\mathbf{5 5}$ & 22.96 & 25.73 & $\mathbf{2 . 7 7}$ & $\mathbf{1 2 . 0 6}$ \\
$\mathbf{6 0}$ & $\mathbf{2 5 . 1 9}$ & 30.00 & 4.81 & $\mathbf{1 9 . 0 8}$ \\
$\mathbf{6 5}$ & $\mathbf{2 6 . 6 5}$ & 30.00 & 3.35 & 12.57 \\
70 & 27.53 & $\mathbf{3 0 . 0 0}$ & 2.47 & 8.98 \\
75 & 28.02 & 30.00 & 1.98 & 7.06 \\
\hline
\end{tabular}

premium is positive, that is, the value of an American option exceeds its European counterpart. Table 1 illustrates the magnitude of the early exercise premium for the previous parameter values. As shown in the last two columns of Table 1, the early exercise premium first increases and then decreases as the initial stock price rises. When the stock price is near the cap, the early exercise premium is nearly 20 percent of the value of the European option.

\subsection{Hedging American capped call options}

The valuation formulas derived in the article are important to the issuers and holders of capped call options not only for pricing but also for hedging. The valuation formulas permit a straightforward and 
Table 2

Hedge ratios $(\theta C / B S)$

\begin{tabular}{lcccc}
\hline $\begin{array}{c}\text { Stock } \\
\text { price }\end{array}$ & $\begin{array}{c}\text { European } \\
\text { uncapped } \\
\text { ( })\end{array}$ & $\begin{array}{c}\text { American } \\
\text { capped } \\
\text { (in) }\end{array}$ & $\begin{array}{c}\text { American capped } \\
\text { delayed exercise } \\
\text { (iii) }\end{array}$ & $\begin{array}{c}\text { European } \\
\text { capped } \\
\text { (iv) }\end{array}$ \\
\hline $\mathbf{3 5}$ & 0.87 & 0.81 & 0.87 & $\mathbf{0 . 8 6}$ \\
$\mathbf{4 0}$ & 0.96 & 0.96 & 0.95 & $\mathbf{0 . 9 2}$ \\
$\mathbf{4 5}$ & 0.99 & $\mathbf{0 . 9 7}$ & 0.94 & 0.85 \\
$\mathbf{5 0}$ & 1.00 & $\mathbf{0 . 9 4}$ & 0.82 & 0.71 \\
$\mathbf{5 5}$ & 1.00 & $\mathbf{0 . 8 9}$ & 0.61 & 0.53 \\
$\mathbf{6 0}$ & 1.00 & $\mathbf{0 . 0 0}$ & $\mathbf{0 . 3 8}$ & 0.36 \\
$\mathbf{6 5}$ & $\mathbf{1 . 0 0}$ & $\mathbf{0 . 0 0}$ & $\mathbf{0 . 1 9}$ & $\mathbf{0 . 2 3}$ \\
$\mathbf{7 0}$ & $\mathbf{1 . 0 0}$ & $\mathbf{0 . 0 0}$ & $\mathbf{0 . 0 8}$ & $\mathbf{0 . 1 3}$ \\
$\mathbf{7 5}$ & 1.00 & $\mathbf{0 . 0 0}$ & $\mathbf{0 . 0 3}$ & 0.07 \\
\hline
\end{tabular}

*The hedge ratio for $S_{0}=59.99$ is 0.79 . The derivative is discontinuous at $S_{0}=L$.

efficient computation of hedging strategies designed to eliminate the risk inherent in a position in these contracts.

Table 2 illustrates how the hedge ratios, $\partial \boldsymbol{C} / \boldsymbol{\partial S}$, depend on the current stock price, $S_{0}$ The hedge ratios of all four options are similar for $S_{0}<L$. For $S_{0} \gg l$, the hedge ratio of option (i) approaches one, (ii) is zero, and (iii) and (iv) approach zero.

The hedge ratios in Table 2 for the American contract [column (ii) may differ quite significantly from those for the European contract [column (iv)]. For example, when the stock price is $\$ 50$ the respective hedge ratios are 0.94 and 0.71 . If the stock price increases to $\$ 65$, the hedge ratios fall to 0 and 0.23 , respectively. Hence, using hedge ratios based on European formulas as an approximation would leave the hedger exposed to significant risk associated with the fluctuation in the underlying asset value.

\subsection{Valuing caps with a constant growth rate}

We consider an option with parameter values: $g=r=0.10, \sigma=0.05$, $T=1, t=0, K=30, S_{0}=60$, and $L_{0}=60$. Since $g=r$, condition (19) in Theorem 4 implies that $t_{f}^{*}=T$. Figure 4 plots $C_{0}^{L}\left(t_{e}, t^{*}, t_{f}^{*}\right)$ for $t_{e}$ ranging from 0 to $T$. Since $\delta=0, t^{*}=T$. For $\boldsymbol{t}_{\boldsymbol{e}}=0, C_{0}^{\boldsymbol{L}}\left(\boldsymbol{t}_{\boldsymbol{e}}, \boldsymbol{t}^{*}, \boldsymbol{t}_{\boldsymbol{f}}^{*}\right)=30$ because the exercise policy calls for immediate exercise. For $t_{e}=\boldsymbol{T}, \boldsymbol{C}_{0}^{\boldsymbol{L}}\left(\boldsymbol{t}_{\boldsymbol{e}}, \boldsymbol{t}^{*}, \boldsymbol{t}_{\boldsymbol{f}}^{*}\right)$ is the value of a European capped call option with cap $L_{T}=L_{0} e^{g T}$. For these parameters, this gives a value of 31.66. The optimal value of $t_{e}$ is $t^{*}{ }_{e}=0.88$ and the option value is $\boldsymbol{C}_{0}^{L}=C_{0}^{L}\left(\boldsymbol{t}_{e}^{*}, \boldsymbol{t}^{*}, \boldsymbol{t}_{f}^{*}\right)=31.68$. The policy of exercising immediately would result in a loss of more than 5 percent of the value of the option. 


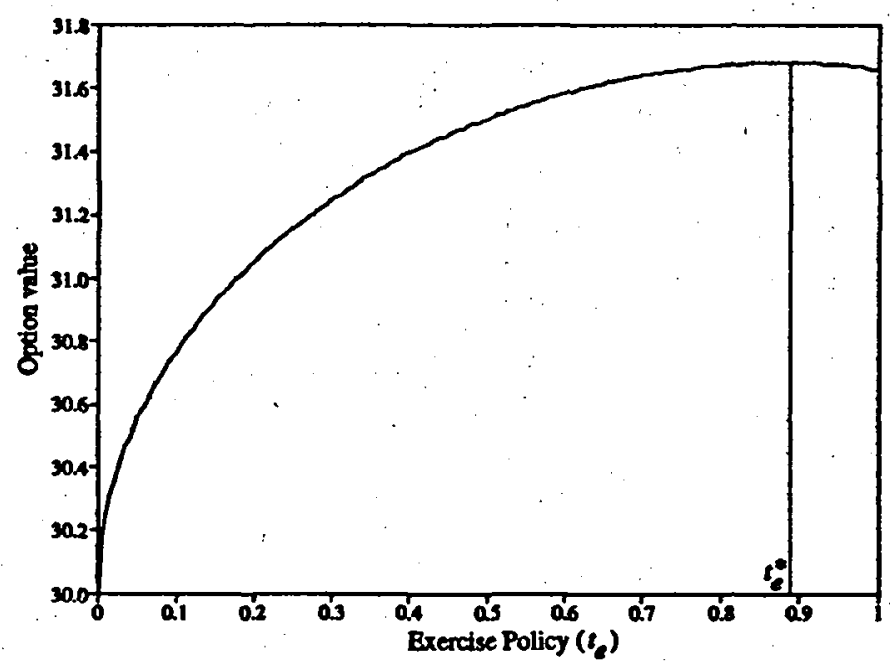

Figure 4

Option value vs. exercise policy

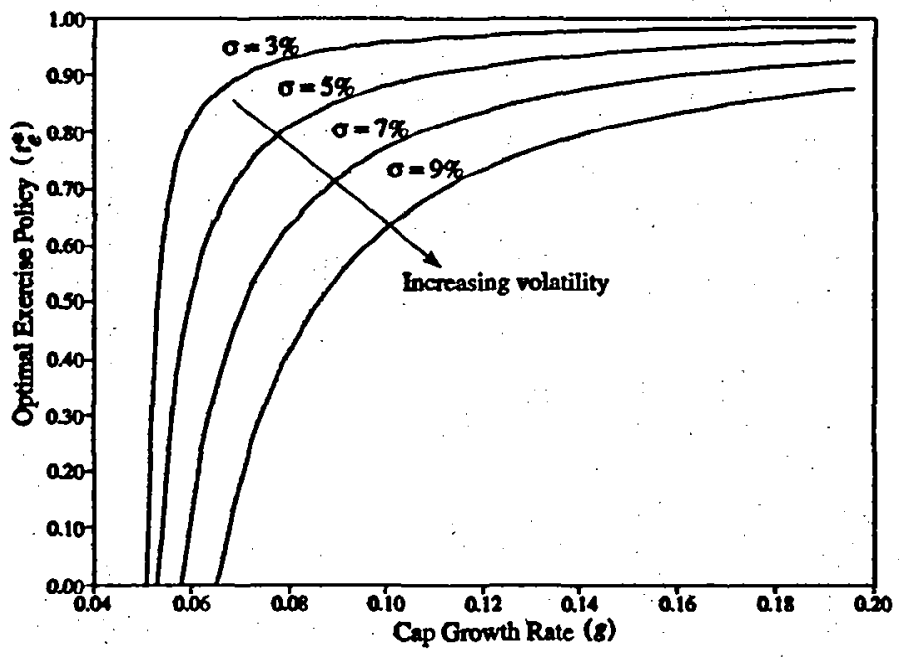

Figure 5

Optimal exercise policy vs. cap growth rate 
Figure 5 shows how $t^{*}{ }_{e}$ varies with the growth rate of the cap, $g$, and with volatility, $\sigma$. The parameter values are the same as in the previous example, except for $g$, which ranges from 0.05 to 0.195 , and $\sigma$, which ranges from 0.03 to 0.09 . In this example, $t^{*}{ }_{e}=0$ for low growth rates and $t^{*}{ }_{e}$ increases as $g$ increases. The volatility of the underlying asset has the opposite effect, that is, $t^{*} e_{e}$ decreases as $\sigma$ increases. For all of these parameter values, $t_{f}^{*}=T$. The parameter $t_{f}$ becomes less than $T$ for smaller values of $g$. For example, suppose that $r=0.05$, $L_{0}=60, K=30$, and $T=1$. Then for $g=0.0255, t_{f}=0.79$. As $g$ decreases, $t_{f}^{*}$ decreases to zero. As $g$ increases, $t_{f}^{*}$ increases to $T$.

\section{Conclusions}

This article focused on the problem of valuing American call options with caps. Since early exercise is allowed, the valuation problem requires the determination of optimal exercise policies. The proof of Theorem 2 showed that early exercise is not optimal whenever the underlying asset's value is below the minimum of the cap and the optimal exercise boundary for the corresponding uncapped option. When the cap is constant and the dividend rate satisfies $\delta \leq r K / L$, it is optimal to exercise at the first time that the price equals or exceeds the cap.

Once the form of the optimal exercise policy is known, a valuation formula for options with delayed exercise periods can be derived. In Section 2, a valuation formula was given for the MILES option contract. A delayed exercise period is one example of a time-varying cap. In Section 3 time varying caps with constant growth rates were analyzed. For these caps, the optimal exercise strategy is given by three endogenous parameters. The exact form of the optimal policy was given in Theorem 4. In part, Theorem 4 says that it is not optimal to exercise prior to time $t^{*}{ }_{e}$ no matter what the value of the underlying asset. This differs. from uncapped call options on dividend paying assets which should be exercised when the value of the underlying asset is sufficiently large.

Computational results were given in Section 4. A comparison of hedge ratios in Table 2 showed similar hedge ratios for capped and uncapped call options when the underlying asset's price is well below the cap. However, as the price increases toward the cap, the hedge ratios differ significantly. For caps with a constant growth rate, optimal exercise parameters $t^{*}{ }_{e}$ and $t_{f}{ }_{f}$ were computed. The dependence of $t^{*}{ }_{e}$ on $g$ and $\sigma$ was also illustrated.

One issue for future analysis is the incorporation of stochastic volatilities into the valuation equations. This may be important in 
view of the empirical evidence which suggests that volatilities are time varying. Another interesting question is the optimality of caps and the design of capped option contracts taking into consideration the interests of the owner and the issuer.

\section{Appendix: Proofs of Theorems}

Proof of Theorem 1. Clearly when $S_{t} \geq L \wedge B_{t}$ immediately exercise is optimal. Now suppose $S_{t}<L \wedge B_{t}$ and $L \geq(r / \delta) K$. In this case there always exists an (uncapped) American option with a shorter maturity $T_{0}$, with $t<T_{0} \leq T$, such that its optimal exercise boundary $B^{0}$ satisfies

See Figure 6 for an illustration. Since early exercise is optimal for this $T_{0}$ maturity option, its value strictly exceeds $S_{t}-K$. From equation (23), this exercise policy is admissible for the capped option. Hence $\boldsymbol{C}_{\boldsymbol{t}}^{\boldsymbol{l}}>\boldsymbol{S} \boldsymbol{\boldsymbol { t }} \boldsymbol{-} \boldsymbol{K}$ and early exercise is suboptimal for the capped option.

Suppose now that $S_{t}<L \wedge B_{t}$ and $L<(r / \delta) K$. In this case, an investor could purchase one call, short one share of stock, and invest

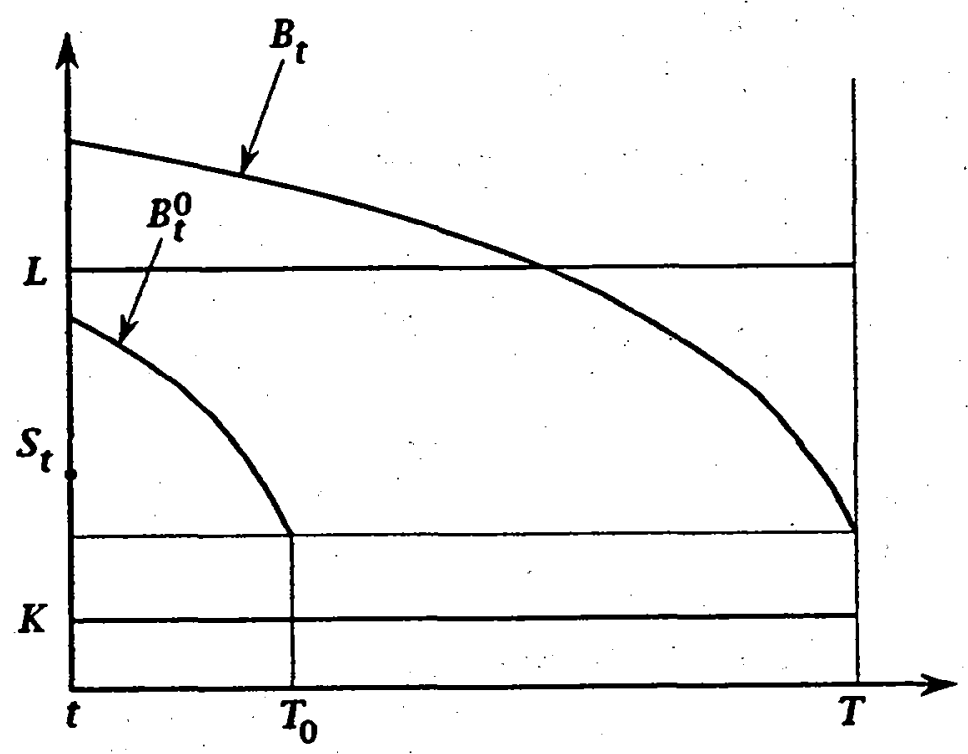

Figure 6

Shorter maturity option boundary 
the proceeds, that is, lend $K$, in the money market account which accrues interest at a rate $r$. If immediate exercise were optimal, then the, total cash flow from these transactions at date $t$ would be zero. Now suppose that the call is exercised at time $\boldsymbol{\tau}$ defined by $\boldsymbol{\tau}_{L} \wedge T$, where $\boldsymbol{\tau}_{\boldsymbol{L}}$ is the hitting time of the cap. Then the net cash flow at time $\tau$ would be

$$
\left(K-S_{\tau}\right) 1_{\left\{S_{i} \leq K\right\}}+\int_{v=t}^{\tau}\left(r K-\delta S_{v}\right) e^{r(\tau-v)} d \nu
$$

Clearly $\left(K-S_{\tau}\right) 1_{\left\{S_{\tau} \leq K\right\}} \geq 0$. Since $S_{v} \leq L<(r / \delta) K$ for $v<\tau$, we have $r K-\delta S_{v}>0$ for $v<\tau$. Hence the integral $\int_{v=t}^{\tau}(r K-$ $\left.\delta \mathcal{S}_{v}\right) \boldsymbol{e}^{r(\tau-\nu)} d v>0$ (probability a.s.). Thus for the value of the call to be consistent with no-arbitrage, immediate exercise at time $t$ cannot be optimal. That is, $\boldsymbol{C}_{\boldsymbol{t}}^{L}>\boldsymbol{S}_{\boldsymbol{t}}-\boldsymbol{K}$.

To prove Corollary 1, Theorem 2, Corollary 2, and later results for caps with constant growth rates, we first state three auxiliary lemmas. These lemmas characterize first-passage times of sets with exponential boundaries when the asset price follows a geometric Brownian motion process. The results complement those of Black and Cox (1976) who characterize the first passage time of the set $[0, L]$ when the stock price starts above the cap, i.e., $S_{t}>L$. Equation (24) below can be derived from equation (7) on p. 356 of Black and Cox (1976) by an appropriate change of variables.

Lemma 1. Suppose $S_{t}$ satisfies the stochastic differential equation

$$
d S_{t}=S_{t}\left[(r-\delta) d t+\sigma d W_{\ell}\right]
$$

For $A \leq B$, define $U\left(S_{t}, A, B, t, T\right)$ to be $P\left[S_{T} \leq A\right.$ and $S_{v}<B$ for $v \in[t, T)]$. Then

$$
U\left(S_{t}, A, B, t, T\right)=N(d(A))-\left(\frac{S_{t}}{B}\right)^{1-2(r-\delta) / \sigma^{2}} N(d(A, B))
$$

when $S_{t} \leq B$ and $t \in[0, T]$, where

$$
d(A)=\frac{1}{\sigma \sqrt{T-t}}\left[\log (A)-\log \left(S_{t}\right)+\left(\delta-r+\frac{1}{2} \sigma^{2}\right)(T-t)\right]
$$




$$
\begin{aligned}
d(A, B)=\frac{1}{\sigma \sqrt{T-t}}\left[\log \left(S_{t}\right)\right. & +\log (A)-2 \log (B) \\
& \left.+\left(\delta-r+\frac{1}{2} \sigma^{2}\right)(T-t)\right]
\end{aligned}
$$

Lemma 2. Suppose that the cup is given by $L_{t}=L_{0} e^{g t}$ and $\boldsymbol{\tau}_{\boldsymbol{L}}$ represents the first time at which $S_{t}$ reaches $L_{t}$. The distribution of the first passage time is

$$
P\left[\tau_{L} \geq T\right]= \begin{cases}N\left(-\bar{d}_{2}^{+}\right)-\lambda_{t}^{1+2(g+\delta-r) / \sigma^{2}} N\left(-\bar{d}_{2}^{-}\right) & \text {if } S_{t} \leq L_{t} \\ N\left(\bar{d}_{2}^{+}\right)-\lambda_{t}^{1+2(g+\delta-r) / \sigma^{2}} N\left(\bar{d}_{2}^{-}\right) & \text {if } S_{t} \geq L_{t}\end{cases}
$$

where $\lambda_{t}=S_{t} / L_{t}, \bar{b}=g+\delta-r+\frac{1}{2} \sigma^{2}$, and $\bar{d}_{2}^{ \pm}=\left[ \pm \log \left(\lambda_{t}\right)-\bar{b}(T-t)\right] /$ $(\sigma \sqrt{T-t})$.

Lem ma 3. The density of the first passage time $\boldsymbol{\tau}_{\boldsymbol{L}}$ is

$$
\gamma(y)= \begin{cases}-n\left(\bar{d}_{2}^{+}\right) \frac{\log \left(\lambda_{t}\right)}{\sigma y^{3 / 2}} & \text { if } S_{t} \leq L_{t} \\ n\left(\bar{d}_{2}^{+}\right) \frac{\log \left(\lambda_{t}\right)}{\sigma y^{3 / 2}} & \text { if } S_{t} \geq L_{t} .\end{cases}
$$

The next lemma summarizes a useful integral of the first passage time density. The integral is parameterized by a constant $a$, which appears in expressions for $\boldsymbol{f}(\boldsymbol{a}), \boldsymbol{\phi}(\boldsymbol{a})$, and $\boldsymbol{\alpha}(\boldsymbol{a})$ defined in Lemma 4. Elsewhere in the article, when $f, \phi$, and $\alpha$ appear without an argument, they refer to $f(0), \phi(0)$, and $\alpha(0)$, respectively.

\section{Lemma 4.}

$$
\begin{aligned}
& \int_{0}^{t} e^{(a-r) y} \gamma(y) d y \\
& \cdots=\left\{\begin{array}{ccc}
\lambda_{0}^{2 \phi(a) / \sigma^{2}} N\left(d_{0}(a)\right) & +\lambda_{0}^{2 \alpha(a) / \sigma^{2}} N\left(d_{0}(a)\right. & \\
& +2 f(a) \sqrt{t} / \sigma) & \\
& & \\
\lambda_{0}^{2 \phi(a) / \sigma^{2}} N\left(-d_{0}(a)\right) & +\lambda_{0}^{2 \alpha(a) / \sigma^{2}} N\left(-d_{0}(a)\right. \\
& -2 f(a) \sqrt{t} / \sigma) & \text { if } \lambda_{0} \geq 1
\end{array}\right.
\end{aligned}
$$

where $d_{0}(a)=\left[\log \left(\lambda_{0}\right)-f(a) t\right] /(\sigma \sqrt{t}), \lambda_{0}=S_{0} / L_{0}, \bar{b}=g+\delta-r+\frac{1}{2} \sigma^{2}$, $f(a)=\left(\bar{b}^{2}+2(r-a) \sigma^{2}\right)^{1 / 2}, \phi(a)=\frac{1}{2}(\bar{b}-f(a))$, and $\alpha(a)=\frac{1}{2}(\bar{b}+$ $f(a))$.

Proof of Corollary 1. The optimal exercise boundary $\boldsymbol{L} \wedge \boldsymbol{B}_{\infty}$ is immediate from Theorem 1. For $S_{t} \geq L \wedge B_{\infty}$, immediate exercise is 
optimal and the option value is $\left(S_{t} \wedge L\right)-K$. For $S_{t}<L \wedge B_{\infty}$, the value of the option can be written as

$$
C_{t}^{L}\left(S_{t}, K, \infty\right)=\left(L \wedge B_{\infty}-K\right) E_{t}^{*}\left[e^{-r(\tau-t)}\right],
$$

where $\boldsymbol{\tau}$ is the hitting time of $\boldsymbol{L} \wedge \boldsymbol{B}_{\infty}$. The risk-neutral valuation equation (25) follows from the optimal exercise boundary and standard pricing results [see Harrison and Kreps (1979)]. The expectation can be written as

$$
\begin{aligned}
E_{t}^{*}\left[e^{-r(\tau-t)}\right] & =\int_{y=0}^{\infty} e^{-r y} \gamma(y) d y \\
& =\lim _{r \uparrow \infty}\left[\lambda_{t}^{2 \phi / \sigma^{2}} N\left(d_{0}\right)+\lambda_{t}^{2 \alpha / \sigma^{2}} N\left(d_{0}+2 f \sqrt{(T-t)} / \sigma\right)\right] \\
& =\left(\frac{S_{t}}{L \wedge B_{\infty}}\right)^{2 \alpha / \sigma^{2}}
\end{aligned}
$$

The second equality follows from Lemma 4 with $a=g=0$ and $\lambda_{t}=S_{t} /\left(L \wedge B_{\infty}\right)$. This proves equation (2).

If there are no dividends, $\boldsymbol{B}_{\infty}=\boldsymbol{\alpha}, \boldsymbol{\alpha}=\boldsymbol{\sigma}^{\mathbf{2}} / \mathbf{2}$, and equation (3) follows directly from equation (2). Alternatively, for $\delta=0$, the discounted stock price is a martingale, and by the optional sampling theorem, $\boldsymbol{S}_{\boldsymbol{t}}=\boldsymbol{E}_{\boldsymbol{t}}^{*}\left[\boldsymbol{e}^{-\boldsymbol{r}(\boldsymbol{\tau}-\boldsymbol{t})} S_{\boldsymbol{\tau}}\right]$ for any stopping time $\boldsymbol{\tau}$. Taking $\boldsymbol{\tau}$ to be the hitting time of the cap $L$ gives $S_{t}=L E_{t}^{*}\left[e^{-r(\tau-t)}\right]$.Substituting this formula into equation (25) gives equation (3).

Proof of Theorem 2. By Theorem 1, for $S_{t} \geq L \wedge B_{t}$ immediate exercise is optimal and the option value is $\left(S_{t} \wedge \boldsymbol{L}\right)-\boldsymbol{K}$. For $\boldsymbol{S}_{\boldsymbol{t}}<\boldsymbol{L} \wedge \boldsymbol{B}_{\boldsymbol{t}}$ and $t \geq t^{*}$, optimal exercise occurs at the first time the boundary $B$ is reached or at maturity, so the capped option value is the standard uncapped option value $C_{t}\left(S_{t}\right)$. For $S_{t}<L \wedge B_{t}$ and $t<t^{*}$ the valuation formula (4) is immediate from Theorem 1.

To obtain (5), first define $S^{n}$ to be the process which is followed by $S$ in the absence of dividends. That is, the processes $S$ and $S^{n}$ are related by $\boldsymbol{S}_{\boldsymbol{t}}^{n}=\boldsymbol{S}_{t} e^{\boldsymbol{s t}}$. This can be used to compute the first expectation in equation (4). When $t^{*}>t$ we have

$$
\begin{aligned}
E_{t}^{*}\left[e^{-r\left(\tau_{L}-t\right)}\left(S_{\tau_{L}}-K\right) 1_{\left\{z_{L}<t\right]}\right]= & \int_{y=0}^{r-t} e^{-r y}(L-K) \gamma(y) d y \\
= & (L-K)\left[\lambda_{t}^{2 \phi / \sigma^{2}} N\left(d_{0}\right)\right. \\
& \left.+\lambda_{t}^{2 \alpha / \sigma^{2}} N\left(d_{0}+2 f \sqrt{t^{*}-t} / \sigma\right)\right] .
\end{aligned}
$$


in (26), $\gamma(\gamma)$ represents the density of the first passage time of $S_{t}$ to the level $L$. The formula for $\gamma(\gamma)$ is given. in Lemma 3 for the case $S_{t} \leq L$ and $g=0$. Equation (27) follows from (26) using Lemma 4 with $a=g=0$.

The second expectation can be written as

$$
\begin{aligned}
E_{t}^{*}\left[e^{-r\left(r^{*}-t\right)} C_{r}\left(S_{r}\right) 1_{\left\{\tau_{L} \geq r\right]}\right]= & e^{-r(r-t)} \int_{x=0}^{L} C_{r}(x) d P\left[S_{r} \leq x, S_{\nu}<L\right. \\
& \text { for } \left.v \in\left[t, t^{*}\right]\right] d x \\
= & e^{-r(r-t)} \int_{x=0}^{L} C_{r}(x) u\left(x, t, t^{*}\right) d x
\end{aligned}
$$

The formula for $u\left(x, t, t^{*}\right)$ in equation (6) in the text follows by differentiating $U\left(S_{t}, x, L, t, t^{*}\right)$ with respect to $x$ [see equation (24) in Lemma 1].

Proof of Corollary 2. The first term in equation (10) is the same as the term in (5) in Theorem 2 with $t^{*}$ replaced by $T$.

The remaining terms in equation (10) follow from

$$
\begin{aligned}
& E\left[e^{-r(T-t)}\left(S_{T}-K\right)^{+} 1_{\left[\tau_{L} \geq r\right]}\right] \\
&=\int_{x=0}^{L} e^{-r(T-t)}(x-K)^{+} d P\left[S_{T} \leq x \text { and } S_{\nu}<L \text { for } v \in[t, T)\right] \\
&=\int_{x=K}^{L} e^{-r(T-t)}(x-K)\left[n\left(d_{1}(x)\right)-\lambda_{t}^{1-2(r-\delta) / \sigma^{2}} n\left(d_{1}^{+}(x)\right)\right] \\
& \times \frac{1}{x \sigma \sqrt{T-t}} d x \\
&= \int_{x=K}^{L} e^{-r(T-t)} \frac{1}{\sigma \sqrt{T-t}}\left[n\left(d_{1}^{-}(x)\right)-\lambda_{t}^{-2(r-\delta) / \sigma^{2}} n\left(d_{1}^{+}(x)\right)\right] d x \\
&-K e^{-r(T-t)}\left[N\left(d_{1}^{-}(L)\right)-N\left(d_{1}^{-}(K)\right)\right. \\
&-\lambda_{t}^{1-2(r-\delta) / \sigma^{2}}\left\{N\left(d_{1}^{+}(L)\right)-N\left(d_{1}^{+}(K)\right)\right] .
\end{aligned}
$$

A simplification of the integral in the previous line gives equation (10) and proves Corollary 2 .

Proof of Theorem 3. The event $\left\{S_{t e} \geq L\right\}$ is equivalent to the event

$$
\left\{z \geq\left[\log \left(L / S_{t}\right)-\left(r-\delta-\frac{1}{2} \sigma^{2}\right)\left(t_{e}-t\right)\right] \frac{1}{\sigma \sqrt{t_{e}-t}}\right\} .
$$


Proof of Theorem 4. We start by considering whether it is optimal to exercise above the cap. At time 0 , the perfect. foresight value of exercising the option at time $s$ (when $S_{s}>L_{s}$ ) is

$$
f(s) \equiv e^{-r s}\left[L_{0} e^{g s}-K\right]
$$

For a trajectory such that $S_{t} \geqq L_{t}$ for all $0 \leq t \leq T$, the optimal exercise time is given by $\hat{\boldsymbol{t}} \equiv \operatorname{argmax}_{0 \leq s \leq T}$. In fact, this $\hat{\boldsymbol{t}}$ is $\boldsymbol{t}_{\boldsymbol{f}}^{*}$, as shown next.

Suppose that $s<T$ and $S_{s}>L_{s}$. There exists a random variable $\boldsymbol{\epsilon}(\boldsymbol{\omega})$ such that $\boldsymbol{S}_{\boldsymbol{s + \epsilon ( \omega )}}>\boldsymbol{L}_{\boldsymbol{s + \epsilon}(\boldsymbol{\omega})}$ (probability a.s.). Hence, it pays to delay exercise if $f^{\prime}(s)>0$. Defining $\boldsymbol{t}_{f}^{*}=\operatorname{argmax}_{0 \leq s \leq T} f(s)$, it is easy to show that $f^{\prime}(s)>0$ for $s<t_{f}^{*}$ and $f^{\prime}(s)<0$ for $s>t^{*}{ }_{f}$. It follows that it does not pay to exercise if $S_{t}>L_{t}$ and $t<t^{*}$. On the other hand, immediate exercise is optimal if $S_{t} \geq L_{t}$ and $t \geq t^{*}{ }_{f}$.

The same argument as in the proof of Theorem 1 shows that it is not optimal to exercise at any time $t<T$ when $S_{t}<L_{t} \wedge B_{t}$. It also shows that exercise is optimal whenever $t \geq t^{*}$ and $B t \leq S_{t} \leq L_{t}$.

Hence, exercise is only optimal at time $t<\boldsymbol{t}^{*} \wedge \boldsymbol{t}_{f}^{*}$ if $\boldsymbol{S}_{\boldsymbol{t}}=\boldsymbol{L}_{\boldsymbol{t}}$. To show that a $\left(t_{e}, t^{*}, t_{f}\right)$ exercise policy is optimal, it remains to be shown that the optimal exercise set at the cap is connected and extends for some $t_{e}$ to $t^{*} \wedge t^{*}{ }_{f}$ Lemma 5, which follows, asserts exactly this result and hence proves Theorem 4 .

Lemma 5 (Connectedness of the exercise set). Suppose that it is optimal to exercise at time $t_{l}$ when $S_{t 1}=L_{t 1}$. Then for all times $t_{2}$ satisfying $t_{l} \leq t_{2} \leq t^{*} \wedge t^{*}{ }^{*}$, it is optimal to exercise when $S_{t e}=L_{t 2}$.

Proof of Lemma 5. Without loss of generality, we assume that $t^{*}{ }_{f}=$ $T$. Let $\boldsymbol{\tau}(L, t, T)$ denote the optimal time to exercise an option with a cap of L, starting at time $t$, which matures at time $T$. Let $C_{t}^{r(L, t, T)}(S, L, T)$ denote the option price under the policy $\boldsymbol{\tau}(L, t, T)$ when the stock price at time $t$ is $S$, the cap function is $L$, and the option matures at time $\frac{T}{2}$. Define the cap $\bar{L}$ by $\bar{L}_{t}=L_{t+t_{L}-h_{2}}$ for $t \geq t_{2} .{ }^{9}$ Finally, let $S^{1}=L_{t_{1}}$ and $\overline{S^{2}}=L_{t 2}$. By the definition of $\bar{L}, L_{t_{2}}=S^{1}$. Figure 7 illustrates these definitions.

Clatm 1. $L_{t_{1}}-K \geq C_{h_{2}}^{\tau\left(\bar{L}, h_{2}, T\right)}\left(S^{1}, \bar{L}, T\right)$.

${ }^{9}$ For $\mathrm{t}<t_{2}$ the cap $\overline{\boldsymbol{L}}_{\mathbf{1}}$ is never used. 


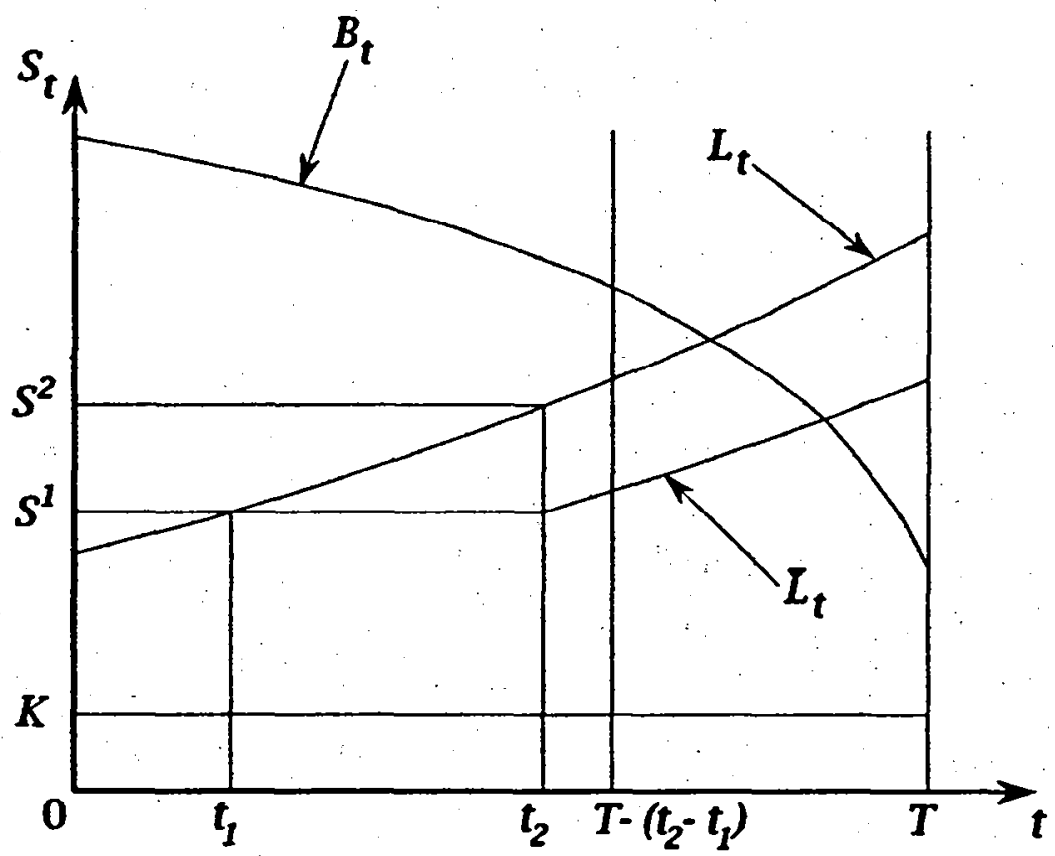

Figure 7

Illustration of the caps $L$ and $Z$

Proof of Claim 1.

$$
\begin{aligned}
& \boldsymbol{L}_{t_{1}}-K=C_{t_{1}}^{\tau\left(L, h_{1}, T\right)}\left(S^{1}, L, T\right) \quad \text { (since it is optimal to exercise at } t_{1} \text { ) } \\
& \leq C_{t_{1}}^{\tau\left(L, t_{1}, T-\left(t_{2}-t_{1}\right)\right)}\left(S^{1}, L, T-\left(t_{2}-t_{1}\right)\right) \\
& =C_{h}^{r\left(\bar{L} h_{2}, T\right)}\left(S^{1}, \bar{L}, T\right)
\end{aligned}
$$

In the second line, $\boldsymbol{\tau}\left(\boldsymbol{L}, \boldsymbol{t}_{1}, \boldsymbol{T}-\left(\boldsymbol{t}_{\mathbf{2}}-\boldsymbol{t}_{1}\right)\right)$ is the optimal exercise policy at time $t_{l}$ for an option with maturity $T-\left(t_{2}-t_{1}\right)$ and cap $L$. Since the cap $L$ between $t_{1}$ and $T-\left(t_{2}-t_{1}\right)$ is same as the cap $\overline{\boldsymbol{L}}$ between $t_{2}$ and $T$, the equality in the third line holds.

Clatm 2. $C_{h_{2}}^{\tau\left(L, h_{2} T\right)}\left(S^{2}, L, T\right)-C_{h_{2}\left(\bar{L}, h_{2}, T\right)}\left(S^{1}, \bar{L}, T\right) \leq L_{h_{2}}-L_{t_{1}}$.

Proof of Claim 2.

$$
\begin{aligned}
& C_{b_{2}}^{r\left(L, h_{2}, T\right)}\left(S^{2}, L, T\right)-C_{h_{2}}^{\mathrm{r}\left(\bar{L}_{t_{2}}, T\right)}\left(S^{1}, \tilde{L}, T\right) \\
& \leq C_{h^{2}}^{\tau\left(L, h_{2}, T\right)}\left(S^{2}, L, T\right)-C_{h_{2}}^{\tau\left(L, h_{2}, T\right)}\left(S^{1}, \bar{l}, T\right)
\end{aligned}
$$




$$
\begin{aligned}
& \text { (suboptimality of } \tau \equiv \tau\left(L, t_{2}, T\right) \text { for } C_{t_{2}}^{\tau\left(\tilde{L}_{2}, T\right)}\left(S^{1}, \tilde{L}, T\right) \text { ) }
\end{aligned}
$$

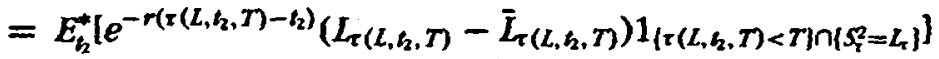

$$
\begin{aligned}
& +E_{h}^{*}\left[e ^ { - r ( \tau ( L , h , T ) \cdot k _ { 2 } ) } \left[\left(S_{\tau\left(L, h_{2}, T\right)}^{2}-K\right)^{+}\right.\right. \\
& \left.-\left(S_{\tau(L, h, T)}^{1}-K\right)^{+}\right] 1_{[\tau(L, h, T)<T) \cap\left[S_{\tau}^{2}=B_{r}\right]} \\
& +E_{\zeta_{2}}^{*}\left[e^{-r\left(T-b_{2}\right)}\left(L_{T}-\bar{L}_{T}\right) 1_{\left\{\tau\left(L, b_{2}, T\right) \geq T\right\} \cap\left\{S_{T}^{2} \geq L_{T}\right\}}\right] \\
& +E_{h^{*}}^{*}\left[e^{-r\left(T-\hbar^{\prime}\right)}\left\{\left(S_{T}^{2}-K\right)^{+}-\left(S_{T}^{1}-K\right)^{+}\right] 1_{\{\tau(L, h, T) \geq T) \cap\left(S_{T}^{2}<L_{T}\right\}}\right] \\
& \text { (where } S_{\tau}^{t}=S^{i} e^{\left(r-\delta-\frac{1}{2} \sigma^{2}\right)\left(\tau-t_{2}\right)+\sigma\left(z_{i}-z_{z_{2}}\right)} \text { for } t=1,2 \\
& \text { and } \left.S_{T}^{i}=S^{i} e^{\left(r-\delta-\frac{1}{2} \sigma^{2}\right)\left(T-r_{2}\right)+\sigma\left(z_{T}-z_{i_{2}}\right)} \text { for } i=1,2\right) \\
& \leq E_{h}^{*}\left[e^{-r\left(\tau\left(L, h_{2}, T\right)-h_{2}\right)}\left(S_{\tau\left(L, h_{2}, T\right)}^{2}-S_{\tau\left(L, h^{2}, T\right)}^{1}\right) 1_{\left\{\tau\left(L, h_{2}, T\right)<T\right)}\right] \\
& +E_{t_{2}}^{+}\left[e^{-r\left(T-b_{2}\right)}\left(S_{T}^{2}-S_{T}^{1}\right) 1_{[r(L, h, T) \geq T) \cap\left(S_{T}^{2} \geq L_{T}\right\}}\right] \\
& +E_{\xi_{2}}^{*}\left[e^{-r\left(T-\xi_{2}\right)}\left(S_{T}^{2}-S_{T}^{1}\right) 1_{\left\{r\left(L, h_{2}, T\right) \geq T\right\} \cap\left(S_{T}^{2}<L_{T}\right\}}\right] \\
& \text { (since on the event } S_{T}^{2} \geq L_{T}, S_{T}^{2}-S_{T}^{1} \geq L_{T}-\bar{L}_{T} \text { ) } \\
& \leq S^{2}-S^{1} \quad \text { (see the argument in the text below) } \\
& =L_{t_{2}}-\bar{L}_{t_{2}}=L_{t_{2}}-L_{t_{1}} \text {. }
\end{aligned}
$$

For $\boldsymbol{\tau} \geq \boldsymbol{t}_{\mathbf{2}}$ the quantity $\boldsymbol{S}_{\boldsymbol{\tau}}^{\mathbf{2}}-\boldsymbol{S}_{\boldsymbol{\tau}}^{\mathbf{1}}$ can be written as

$$
\left(S^{2}-S^{1}\right) e^{\left(r-\delta-\frac{1}{2} \sigma^{2}\right)\left(r-b_{2}\right)+\sigma\left(z_{2}-z_{2}\right)}
$$

where $S^{2}>S^{l}$. Since $\delta \geq 0$, the process $\boldsymbol{e}^{-\boldsymbol{r}\left(\boldsymbol{\tau}-\boldsymbol{k}_{\mathbf{2}}\right)}\left(\boldsymbol{S}_{\tau}^{2}-\boldsymbol{S}_{\tau}^{\mathbf{1}}\right)$ is a supermartingale. Hence for any stopping time $\boldsymbol{\tau} \geq \boldsymbol{t}_{\mathbf{2}}$, the last inequality follows.

Combining Claims 1 and 2 gives

$$
C_{t_{2}}^{\tau\left(L, t_{2}, T\right)}\left(S^{2}, L, T\right) \leq L_{t_{2}}-K,
$$

which implies that exercise is optimal at time $t_{2}$ if $S^{2}=L_{t 2}$. This shows that the optimal exercise set is connected and proves Lemma 5.

Proof of Theorem 5. The option value under the $\left(t_{e}, t^{*}, t_{f}\right)$ policy can be written as

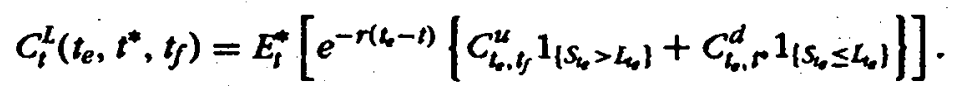

writing $S_{t_{6}}=S_{t} \exp \left[\left(r-\delta-\frac{1}{2} \sigma^{2}\right)\left(t_{e}-t\right)+\sigma z \sqrt{t_{e}-t}\right]$ and $L_{t_{6}}=$ $L_{t} \exp \left[g\left(t_{e}-t\right)\right]$ implies $S_{t_{e}} \geq L_{t}$ if and only if $z \geq-\bar{d}_{2}^{+}\left(\lambda_{t}, t_{e}-t\right)$. Also 
note that $\lambda_{t_{6}}=\lambda_{t} \exp \left[-\bar{b}\left(t_{e}-t\right)+\sigma z \sqrt{t_{e}-t}\right]$. Using these expressions in the expectation above gives equation (15). Expressions for $\boldsymbol{C}_{\boldsymbol{L}, \boldsymbol{h}}^{\boldsymbol{u}}$ and $C_{t, \boldsymbol{t}}^{d}$ are proved in Lemmas 6 and 7, which follow.

Lemma 6. At time $t_{e}$ with $S_{t e}>L_{t e}$, the value of the option corresponding to the $\left(t_{e}, t^{*}, t_{f}\right)$ policy is independent of $t^{*}$. The value, denoted $C_{b_{0}, t_{f}}^{u}$, is given by equation (16) in the statement of Theorem 5.

Proof of Lemma 6.

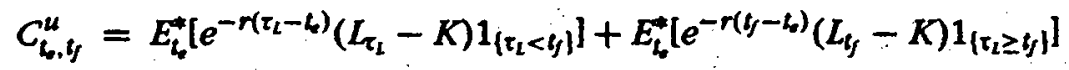

$$
\begin{aligned}
& =L_{L} \int_{y=0}^{y-L_{0}} e^{(g-r) y} \gamma(y) d y \\
& -K \int_{y=0}^{t_{y}-f_{i}} e^{-r y} \gamma(y) d y \\
& +\left[L_{t_{e}} e^{g\left(t_{f}-t_{s}\right)}-K\right] e^{-r\left(t_{f}-t_{0}\right)} E_{t_{f}}^{*}\left[1_{\left.\mid t_{t} \geq t_{f}\right]}\right]
\end{aligned}
$$

Applying Lemma 4 with $a=g$ to the first integral, with $a=0$ to the second integral, and then applying Lemma 2 to the last expectation proves Lemma 6.

Lemma 7. At time $t_{e}$ with $S_{t e} \leqq L_{t e}$, the value of the option under the $\left(t_{e}, t^{*}, t_{f}\right)$ policy, denoted $\boldsymbol{C}_{\mathrm{t} . \mathrm{f}}^{d}$ is given by equation (17) in the statement of Theorem 5.

Proof of Lemma 7.

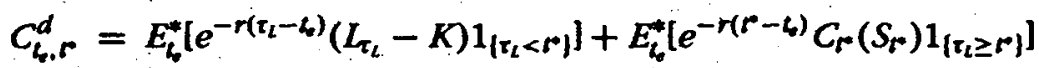

$$
\begin{aligned}
& =L_{t_{0}} \int_{y=0}^{r-t_{0}} e^{(g-r) y} \gamma(y) d y \\
& -K \int_{y=0}^{r-t_{0}} e^{-r y} \gamma(y) d y \\
& +\int_{x=0}^{t_{\infty}} e^{-r\left(r^{*}-t_{s}\right)} C_{r}(x) \bar{u}\left(x, t_{e}, t^{*}\right) d x .
\end{aligned}
$$

The first two integrals can be evaluated using Lemma 4 . The term $\bar{u}\left(\boldsymbol{x}, \boldsymbol{t}_{\boldsymbol{e}}, \boldsymbol{t}^{*}\right)$ represents the density of $S_{t^{*}}$ given that $\boldsymbol{\tau}_{\boldsymbol{L}} \geq \boldsymbol{t}^{*}$. Applying Lemma 1 with a change of variables gives a formula for $P\left[S_{t^{*}} \leq x\right.$ and $S_{v}<L_{v}$ for $\left.\boldsymbol{v} \in\left[\boldsymbol{t}_{\boldsymbol{e}}, \boldsymbol{t}^{*}\right)\right]$. Differentiating with respect to $x$ gives the stated formula for $\bar{u}\left(x, t_{e}, t^{*}\right)$. 
Proof of Theorem 6. The first part of the proof of Theorem 4 shows that $t_{f}^{*}$ is given by

$$
t_{f}^{*}=\underset{0 \leq s \leq r}{\operatorname{argmax}} f(s)
$$

where $f(s)=e^{-r s}\left[L_{0} e^{g s}-K\right]$. The derivative of $f(s)$ is

$$
f^{\prime}(s)=e^{-r s}\left[(g-r) L_{0} e^{g s}+r K\right] .
$$

Equation (28) implies that $f$ is unimodal. If condition (19) holds, i.e., if $(g-r) L_{0} e^{g}+r K \geq 0$, then $f^{\prime}(s) \geq 0$ for all $0 \leq s \leq T$. This means that the marginal value of waiting to exercise is positive, so it is not optimal to exercise above the cap. Hence $t_{f}^{*}=T$.

If condition (20) holds, that is, if $(g-r) L_{0}+r K \leq 0$, then $f^{\prime}(s) \leq 0$ for all $0 \leq s \leq T$. This means that the marginal value of waiting to exercise is negative, so immediate exercise is always optimal at or above the cap. Hence $t_{f}{ }_{f}=0$. Finally, if $0<t_{f}^{*}<T$, then $f^{\prime}\left(t_{f}^{*}\right)=0$, which implies equation (21).

The optimal value of $t_{e}$, denoted $t^{*}{ }_{e}$, is given by the solution of the univariate nonlinear program $(\mathrm{P})$ :

$$
\text { (P) } \max _{t_{e}} c_{0}^{L}\left(i_{e}, t^{*}, t_{f}^{*}\right)
$$

subject to:

$$
0 \leq t_{e} \leq t^{*} \wedge t_{f}^{*}
$$

The Karush-Kuhn-Tucker Theorem gives necessary conditions for $t^{*}$ to solve $(P)$. If the optimal solution to $(P)$ is an interior solution, that is, if $0<t^{*}{ }_{e}<t^{*} \wedge t_{f}^{*}$, then

$$
\left.\frac{\partial C_{0}^{L}\left(t_{e}, t^{*}, t_{f}^{*}\right)}{\partial t_{e}}\right|_{t_{0}=\varepsilon_{e}}=0 .
$$

By the Markovian property of the underlying asset process, the optimal solution to $(P)$ is independent of $S_{0}$. For convenience, we take $S_{0}=L_{0}$, that is, $\lambda_{0}=1$, in equation (29). Taking the partial derivative of the expression for $\boldsymbol{C}_{0}^{L}\left(\boldsymbol{t}_{\boldsymbol{e}}, \boldsymbol{t}^{*}, \boldsymbol{t}_{f}^{*}\right)$ in equation (15) and setting the result to zero at $\mathrm{t}_{e}=t^{*}{ }_{e}$ gives the integral equation (22). If $t^{*}{ }_{e}=t^{*} \wedge t^{*}{ }_{f}$ then $\partial C_{0}^{L}\left(t_{e}, t^{*}, t_{f}^{*}\right) / \partial t_{e} \geq 0$ when evaluated at $t_{e}=t^{*} \wedge t_{f}{ }_{f}$ If $t^{*}{ }_{e}=0$ is the optimal solution to $(P)$, then $\lim _{t_{b}+0} \partial C_{0}^{L}\left(t_{e}, t^{*}, t_{f}^{*}\right) / \partial t_{e} \leq 0$. 


\section{References}

Black, F., and J. C. Cox, 1976, "Valuing Corporate Securities: Some Effects of Bond Indenture Provisions," Journal of Finance, 31, 351-367.

Black, F., and M. Scholes, 1973, "The Pricing of Options and Corporate Liabilities," Journal of Political Economy, 81, 637-654.

Boyle, P. P., and S. H. Law, 1994, "Bumping Up Against the Barrier with the Binomial Method," Journal of Derivatives, 1 , No. 4, 6-14.

Boyle, P. P., and S. M. Turnbull, 1989, "Pricing and Hedging Capped Options," Journal of Futures Markets, 9, 41-54.

Broadie, M., and J. B. Detemple, 1993, “The Valuation of American Capped Call Options,” working paper, Columbia University.

Broadie, M., and J. B. Detemple, 1994, "American Option Valuation: New Bounds, Approximations, and a Comparison of Existing Methods," working paper, Columbia University.

Carr, P., R. Jarrow, and R. Myneni, 1992, “Alternative Characterizations of American Put Options," Mathematical Finance, 2, 87-106.

Cox, J. C., and M. Rubinstein, 1985, Options Markets, Prentice-Hall, Englewood Cliffs, N.J.

Cox, J. C., S. A. Ross, and M. Rubinstein, 1979, “Option Pricing: A Simplified Approach,” Journal of Financial Economics, 7, 229-263.

Flesaker, B., 1992, “The Design and Valuation of Capped Stock Index Options,” working paper, University of Illinois at Urbana-Champaign.

Harrison, J. M., and D. Kreps, 1979, "Martingales and Arbitrage in Multiperiod Securities Markets," Journal of Economic Theory, 20, 381-408.

Kim, I. J., 1990, “The Analytic Valuation of American Options," Review of Financial Studies, 3, $547-572$.

Rubinstein, M., and E. Reiner, 1991, "Breaking Down the Barriers,” Risk, 4, 28-35. 